\title{
World influence and interactions of universities from Wikipedia networks
}

\author{
Célestin Coquidé ${ }^{1 \mathrm{a}}$, José Lages $^{1 \mathrm{~b}}$, and Dima L. Shepelyansky ${ }^{2 \mathrm{c}}$ \\ 1 Institut UTINAM, Observatoire des Sciences de l'Univers THETA, CNRS, Université de Bourgogne Franche-Comté, Besançon, \\ France \\ 2 Laboratoire de Physique Théorique, IRSAMC, Université de Toulouse, CNRS, UPS, 31062 Toulouse, France
}

Received: / Revised version: date

\begin{abstract}
We present Wikipedia Ranking of World Universities (WRWU) based on analysis of networks of 24 Wikipedia editions collected in May 2017. With PageRank and CheiRank algorithms we determine ranking of universities averaged over cultural views of these editions. The comparison with the Shanghai ranking gives overlap of $60 \%$ for top 100 universities showing that WRWU gives more significance to their historical development. We show that the new reduced Google matrix algorithm allows to determine interactions between leading universities on a scale of ten centuries. This approach also determines the influence of specific universities on world countries. We also compare different cultural views of Wikipedia editions on significance and influence of universities.
\end{abstract}

PACS. 89.75.Fb Structures and organization in complex systems - 89.75.Hc Networks and genealogical trees -89.20 .Hh World Wide Web, Internet

\section{Introduction}

The importance of universities for progress of humanity is broadly recognized world wide. Thus in 2017 UNESCO emphasizes the role of universities and higher education institutes in fostering sustainable development and empowering learners [1]. The efficiency of university education gained a high political importance in many world countries. Various tools have been developed to measure quantitatively this efficiency among which the ranking of universities gained significant importance as reviewed in [2]. Thus the Academic Ranking of World Universities (ARWU), compiled by Shanghai Jiao Tong University since 2003 (Shanghai ranking) [3], generated a significant political impact on evaluation of higher education efficiency in many countries [2]. For example, the ARWU stimulated the emergence of LABEX, IDEX projects in France [4] and the Russian Academic Excellence Project [5] with allocation of significant financial supports. In addition to ARWU other international ranking systems of universities appeared (see e.g. $[6,7,8]$ ). Various strong and weak features of ranking methodology are reviewed in $[9$, $10,11]$. Of course, the ranking systems are based on different specific criteria with different cultural preferences of rather larger groups realizing these rankings. Already, the presence of many ranking systems indicates the presence of bias in each of above ranking systems.

\footnotetext{
a email address: celestin.coquide@utinam.cnrs.fr

b email address: jose.lages@utinam.cnrs.fr

c email address: dima@irsamc.ups-tlse.fr
}

Another purely mathematical and statistical approach to ranking of world universities has been developed in [12, $13,14]$ on the basis of Google matrix analysis of Wikipedia networks. For each Wikipedia language edition a network is composed by all Wikipedia articles with directed links between them generated by mutual quotations of a given article to other articles. In [12] the analysis was performed only for English Wikipedia (ENWIKI) of year 2009, other years for ENWIKI were considered in [13], while in [14] this analysis was done with 24 language Wikipedia editions of 2013 that allowed to reduce significantly cultural bias (these 24 networks had been collected and analyzed for historical figures in [15] in the frame of EC FET Open project NADINE [16]). The Google matrix analysis [12, $13,14]$ is based on the PageRank algorithm [17] which detailed description is given in [18]. Some additional characteristics have been also used for description of network nodes (Wikipedia articles), like CheiRank and 2DRank, as described in $[19,20]$. Thus the Wikipedia Ranking of World Universities (WRWU2013) from 24 Wikipedia networks was introduced in [14] and it was shown that its top 100 universities has $62 \%$ overlap with ARWU. In addition WRWU2013 attracted a significant interest world wide (see [21] and various press highlights listed at [22]). Other research groups also start to apply Wikipedia ranking in Wikiometrics [23]. We also note the growing interest to scientific analysis of several language editions of Wikipedia [24].

In this work we extend the WRWU studies started in [14]. The new elements are: we use 24 Wikipedia edi- 
tions collected in May 2017 [25] and also we apply the recently invented reduced Google matrix (REGOMAX) method [26]. This new method allows to determine effective interactions between a selected relatively small subset of network nodes taking into account all pathways between them via the global huge network with millions of nodes. The efficiency of the REGOMAX method has been demonstrated on examples of analysis of interactions of political leaders [27], terror networks [28] and proteinprotein interactions in cancer networks [29]. Here, using the REGOMAX method we obtain effective interactions between a group of selected universities and determine their influence on world countries. The new ranking of universities from Wikipedia 2017 editions is compared with those of 2013.

The paper is composed as follows: Section 2 gives description of network datasets; Section 3 describes Google matrix construction and PageRank, CheiRank algorithms with overview of the reduced Google matrix approach; Section 4 presents results on global ranking of universities from 24 editions and their distribution over world countries; Section 5 provides REGOMAX results for English edition determining the world influence of specific universities; the interactions between top 20 universities are analyzed in Section 6 comparing views of English, French, German and Russian editions; in Section 7 we obtained the reduced Google matrix of top 100 universities averaged over 24 editions and analyze the interactions between universities on the scale of 10 centuries and all continents; discussion of the results is given in Section 8. All detailed ranking results of WRWU2017 are available at [30] and complementary figures and tables are in Supplementary Information (see from p. 18).

\section{Datasets}

We use the datasets of 24 Wikipedia editions extracted in May 2017 [25] (see also [30]). The size of each network is given in Table 1. Compared to 2013 discussed in [15] there is a significant size increase for each edition, especially for Swedish (SV) where a part of articles is now computer generated. The number of links is given at [30]. On average there are about 20 links per node. Self-citation links are not considered (references on the article inside the same article are eliminated).

\section{Description of algorithms and methods}

\subsection{Google matrix, PageRank and CheiRank algorithms}

The mathematical grounds of this study are based on Markov chain theory and, in particular, on the Google matrix analysis initially introduced in 1998 by Google's co-founders, Brin and Page [17], for hypertext analysis of the World Wide Web. Let us consider the network of the $N$ articles of a given Wikipedia edition. The network adjacency matrix element $A_{i j}$ is equal to 1 if article $j$ quotes article $i$ and equal to 0 otherwise. The Google matrix element $G_{i j}=\alpha S_{i j}+(1-\alpha) / N$ gives a transition probability that a random reader jumps from article $j$ to article $i$. The stochastic matrix element $S_{i j}$ is $S_{i j}=A_{i j} / \sum_{i=1}^{N} A_{i j}$ if article $j$ quotes at least one other article, otherwise $S_{i j}=1 / N$. The second term in $G$ proportional to $(1-\alpha)$, where $0.5<\alpha<1$ is the damping factor, allows to a random reader to escape from isolated sets of articles. More details can be found in [18]. Here we use the value $\alpha=0.85$ typical for WWW studies [18]. The Google matrix $G$, constructed as described above, belongs to the class of Perron-Frobenius operators [18]. The eigenvector $\mathbf{P}$ with the largest eigenvalue $\lambda=1$ is the solution of equation $G \mathbf{P}=\mathbf{P}$. This PageRank vector $\mathbf{P}$ has positive or zero components and describes the steady-state probability distribution of the Markov process encoded in the Google matrix $G$. Assuming an infinite random process, the vector component $P_{i}$ is proportional to the number of times a random reader reaches an article $i$. It is convenient to sort the vector components $P_{1}, \ldots, P_{N}$ in descending order: the article associated to the highest (lowest) vector component has the top (last) rank index $K=1(K=N)$. The PageRank algorithm measures the relative influence of articles. Recursively, more an article is quoted by influent articles, more high is its probability.

As proposed in [31] we also consider the same network of articles but with inverted links, i.e., article $j$ points toward article $i$ if article $j$ is quoted by article $i$. This inverted network is defined by the adjacency matrix elements, $A_{i j}^{*}=A_{j i}$, which can be used to build successively the corresponding stochastic matrix elements, $S_{i j}^{*}$, and the corresponding Google matrix elements, $G_{i j}^{*}$. The CheiRank vector $\mathbf{P}^{*}$ is then defined such as $G^{*} \mathbf{P}^{*}=\mathbf{P}^{*}$ and the CheiRank is constructed similarly to the PageRank $[31,12,19]$. The CheiRank algorithm measures the relative communicative ability of the articles. Recursively, the more an articles quotes very communicative articles, the more it is communicative.

The properties of the Google matrix spectrum and eigenstates and their various applications are discussed in detail in $[18,19,20]$.

Table 1. Wikipedia directed networks of 2017 from 24 considered language editions; here $N$ is the number of articles. Wikipedia data were collected in May 2017.

\begin{tabular}{llr|llr}
\hline Edition & Language & $N$ & Edition & Language & $N$ \\
\hline \hline EN & English & 5416537 & ZH & Chinese & 939625 \\
SV & Swedish & 3786455 & FA & Persian & 539926 \\
DE & German & 2057898 & AR & Arabic & 519714 \\
NL & Dutch & 1900222 & HU & Hungarian & 409297 \\
FR & French & 1866546 & KO & Korean & 380086 \\
RU & Russian & 1391225 & TR & Turkish & 291873 \\
IT & Italian & 1353276 & MS & Malaysian & 289234 \\
ES & Spanish & 1287834 & DA & Danish & 225523 \\
PL & Polish & 1219733 & HE & Hebrew & 205411 \\
VI & Vietnamese & 1155932 & EL & Greek & 130429 \\
JP & Japanese & 1058950 & HI & Hindi & 121503 \\
PT & Portuguese & 967162 & TH & Thai & 116495 \\
\hline
\end{tabular}




\subsection{The reduced Google matrix}

The concept of reduced Google matrix (REGOMAX) was introduced in [26] and tested with Wikipedia networks in $[27,28]$ and protein-protein networks [29]. The method is based on the construction of a Google matrix for a relatively small subset of nodes embedded into a much larger network taking into account all indirect interactions between subset nodes via the remaining huge part of the network.

Let us consider a small subset $\mathcal{S}_{\mathrm{r}}$ of $n_{\mathrm{r}} \ll N$ articles, and the complementary subset $\mathcal{S}_{\mathrm{s}}$ of the $n_{\mathrm{s}}=N-n_{\mathrm{r}} \simeq N$ remaining articles. For convenience, the Google matrix can be rewritten as

$$
G=\left(\begin{array}{ll}
G_{\mathrm{rr}} & G_{\mathrm{rs}} \\
G_{\mathrm{sr}} & G_{\mathrm{ss}}
\end{array}\right)
$$

where the submatrix $G_{\mathrm{rr}}$, of size $n_{\mathrm{r}} \times n_{\mathrm{r}}$, encodes the transitions between articles of the subset $\mathcal{S}_{\mathrm{r}}$, the submatrix $G_{\mathrm{ss}}$, of size $n_{\mathrm{s}} \times n_{\mathrm{s}}$, encodes the transitions between articles of the subset $\mathcal{S}_{\mathrm{s}}$, the submatrix $G_{\mathrm{rs}}$, of size $n_{\mathrm{r}} \times n_{\mathrm{s}}$, encodes the transitions from articles of the subset $\mathcal{S}_{\mathrm{S}}$ toward articles of the subset $\mathcal{S}_{\mathrm{r}}$, the submatrix $G_{\mathrm{sr}}$, of size $n_{\mathrm{S}} \times n_{\mathrm{r}}$, encodes the transitions from articles of the subset $\mathcal{S}_{\mathrm{r}}$ toward articles of the subset $\mathcal{S}_{\mathrm{s}}$. Since $G \mathbf{P}=\mathbf{P}$, the PageRank vector can be rewritten as

$$
\mathbf{P}=\left(\begin{array}{l}
\mathbf{P}_{\mathrm{r}} \\
\mathbf{P}_{\mathrm{s}}
\end{array}\right)
$$

where the vector $\mathbf{P}_{\mathrm{r}}\left(\mathbf{P}_{\mathrm{s}}\right)$ of size $n_{\mathrm{r}}\left(n_{\mathrm{S}}\right)$ contains the PageRank vector components associated to articles of the $\mathcal{S}_{\mathrm{r}}$ $\left(\mathcal{S}_{\mathrm{S}}\right)$ subset. The reduced Google matrix $G_{\mathrm{R}}$ associated to articles of the subset $\mathcal{S}_{\mathrm{r}}$ is the $n_{\mathrm{r}} \times n_{\mathrm{r}}$ matrix defined implicitly by the following relation $G_{\mathrm{R}} \mathbf{P}_{\mathrm{r}}=\mathbf{P}_{\mathrm{r}}$. After some algebra, the reduced Google matrix can be written as $[26$, $27,29]$

$$
G_{\mathrm{R}}=G_{\mathrm{rr}}+G_{\text {ind }} \text { where } G_{\text {ind }}=G_{\mathrm{rs}}\left(1_{\mathrm{s}}-G_{\mathrm{ss}}\right)^{-1} G_{\mathrm{sr}} \text {. }
$$

Here $1_{\mathrm{s}}$ is the $n_{\mathrm{s}} \times n_{\mathrm{s}}$ identity matrix. The reduced Google matrix $G_{\mathrm{R}}$ is composed by the $G_{\mathrm{R}}$-submatrix $G_{\mathrm{rr}}$ which encodes the direct links (direct quotations) between the $n_{\mathrm{r}}$ articles of the $\mathcal{S}_{\mathrm{r}}$ subset and by an additional scattering term $G_{\text {ind }}$ which quantifies the indirect links between articles. If there is no direct link from article $j \in \mathcal{S}_{\mathrm{r}}$ to article $i \in \mathcal{S}_{\text {r }}$, i.e., $A_{i j}=0$, then the corresponding $G_{\text {rr }}$ element will be minimum $\left(G_{\mathrm{rr}_{i j}} \sim 1 / N \sim 10^{-7}\right.$ for the May 2017 English Wikipedia network). Conversely, the corresponding $G_{\text {ind }}$ element can be very high highlighting the fact that two articles can be strongly indirectly linked through successive direct links between articles of the $\mathcal{S}_{\mathrm{s}}$ subset (eg, $\left.j \in \mathcal{S}_{\mathrm{r}} \rightarrow k_{1} \in \mathcal{S}_{\mathrm{s}} \rightarrow k_{2} \in \mathcal{S}_{\mathrm{s}} \rightarrow \cdots \rightarrow k_{n} \in \mathcal{S}_{\mathrm{s}} \rightarrow i \in \mathcal{S}_{\mathrm{r}}\right)$. The PageRank vector of $G_{\mathrm{R}}$ has the same components of $n_{r}$ nodes as in the global matrix $G$ (up to a constant normalization factor). The reduced Google matrix $G_{R}$, which conserves the global Google matrix PageRank hierarchy between the $n_{\mathrm{r}}$ articles of the $\mathcal{S}_{\mathrm{r}}$ subset, encodes direct links and effective indirect links between articles. The direct calculation of $G_{\text {ind }}$ converges very slowly since the matrix $\left(1_{\mathrm{s}}-G_{\mathrm{Ss}}\right)^{-1}$ is almost singular, indeed as $n_{\mathrm{r}} \ll n_{\mathrm{s}}$,
$G_{\mathrm{ss}} \sim G$, the leading eigenvalue of $G_{\mathrm{ss}}$ is $\lambda_{c} \sim 1$. Let us associate to the eigenvalue $\lambda_{c}$ the right eigenvector $\boldsymbol{\Psi}_{\mathrm{R}}$ and the left eigenvector $\boldsymbol{\Psi}_{L}$ such as $G_{\mathrm{ss}} \boldsymbol{\Psi}_{\mathrm{R}}=\lambda_{c} \boldsymbol{\Psi}_{\mathrm{R}}$ and $\boldsymbol{\Psi}_{L}^{T} \boldsymbol{\Psi}_{\mathrm{R}}=1$. To speed up calculations, we follow the same procedure as in $[26,27,29]$, splitting the term $\left(1_{\mathrm{s}}-G_{\mathrm{SS}}\right)^{-1}$ in a term $\boldsymbol{\Psi}_{\mathrm{R}} \boldsymbol{\Psi}_{L}^{T}\left(1-\lambda_{c}\right)^{-1}$ which is a projection onto the subspace associated to $\lambda_{c}$ and a term $\left(1_{\mathrm{s}}-\boldsymbol{\Psi}_{\mathrm{R}} \boldsymbol{\Psi}_{L}^{T}\right)\left(1_{\mathrm{s}}-G_{\mathrm{ss}}\right)^{-1}$ which is a projection onto the complementary subspace. This procedure enables us to rewrite the reduced Google matrix as

$$
G_{\mathrm{R}}=G_{\mathrm{rr}}+G_{\mathrm{pr}}+G_{\mathrm{qr}}
$$

where $G_{\mathrm{pr}}=G_{\mathrm{rs}} \boldsymbol{\Psi}_{\mathrm{R}} \boldsymbol{\Psi}_{L}^{T} G_{\mathrm{sr}}\left(1-\lambda_{c}\right)^{-1}$ encodes essentially already known information concerning the PageRank (since $\left.\boldsymbol{\Psi}_{\mathrm{R}} \sim \mathbf{P}\right)$ and $G_{\mathrm{qr}}=G_{\mathrm{rs}}\left(1_{\mathrm{s}}-\boldsymbol{\Psi}_{\mathrm{R}} \boldsymbol{\Psi}_{L}^{T}\right)\left(1_{\mathrm{s}}-G_{\mathrm{ss}}\right)^{-1} G_{\mathrm{sr}}$ encodes hidden interactions between articles which appear due to indirect links via the global network [26,27,29]. In the following we perform analysis of the three components present in (4), we also consider the matrix component $G_{\mathrm{qrnd}}$ obtained from $G_{\mathrm{qr}}$ by taking out the diagonal terms since the self-citations are not very interesting.

\section{Wikipedia Ranking of World Universities from 24 Wikipedia editions of 2017}

Once the articles of 24 considered editions are ranked using PageRank and CheiRank algorithms, we extract for each edition the top 100 articles devoted to institutions of higher education and research. We also consider 2DRank which is a combination of PageRank and CheiRank (see $[12,14]$; 2DRank results are available at [30]). As in [15, 14], from these 24 top 100 listings we obtain the following cumulative score for a given university $U$

$$
\Theta_{U, A}=\sum_{E}\left(101-R_{U, E, A}\right)
$$

where $A$ denotes the algorithm used for the ranking (PageRank or CheiRank or 2DRank), $E$ the Wikipedia edition, $R_{U, E, A}$ the rank of university $U$ in the top 100 universities obtained using algorithm $A$ from edition $E$ of Wikipedia. If an university $U^{\prime}$ is absent from the top 100 universities obtained from an edition $E^{\prime}$ with an algorithm $A^{\prime}$ then we artificially set $R_{U^{\prime}, E^{\prime}, A^{\prime}}=101$. We use ISO 3166-1 alpha-2 country codes [32] (all the used country codes are available at [30]).

The top 10 universities from WRWU2017 with PageRank algorithm and from ARWU2017 are given in Tab. 2 and Tab. 3 respectively. The top 3 places of WRWU are occupied by Oxford, Cambridge and Harvard while for ARWU it is Harvard, Stanford and Cambridge. The universities with significantly lower positions in WRWU (compared to ARWU) are MIT, Berkeley and Caltech, while Oxford significantly improves its position at WRWU going to the first place from 7 th at ARWU.

The overlap between different rankings are presented in Fig. 1. For the top 100 universities we have $60 \%$ overlap 



Fig. 1. Left panel: Overlap $\eta(j)=j_{c} / j$ of ARWU2017 with WRWU2017 as a function of the rank $j$ of WRWU2017. Here $j_{c}$ gives the number of common universities among the first $j$ universities of the two rankings. The color curves show the overlap of ARWU2017 with WRWU2017 (black curve), of ARWU2013 with WRWU2013 (red curve), of ARWU2013 with ARWU2017 (green curve), and of WRWU2013 with WRWU2017 (blue curve). Right panel: Overlap of ARWU2017 with ENWRWU2017 (black curve), of ARWU2017 with FRWRWU2017 (red curve) and of ARWU2017 with DEWRWU2017 (blue curve). The horizontal axis label $j$ is the rank of ARWU2017.

between WRWU PageRank list and ARWU list in 2017. For 2013 this overlap was slightly higher at $62 \%$. The overlap between WRWU2017 list and WRWU2013 list is 91\% and between ARWU2017 list and ARWU2013 list is $84 \%$. WRWU appears to be stable, top10s in 2013 (Tab. 2) and 2017 (Tab. 3 in [14]) contain the same universities but with some changes in places: Oxbridge keep the two first places but Oxford supersedes Cambridge at the first place; Yale $(9 \rightarrow 5)$ and Chicago $(7 \rightarrow 6)$ improve their ranking whereas Princeton $(5 \rightarrow 7)$ and MIT $(6 \rightarrow 9)$ recede; Harvard $(3 \rightarrow 3)$, Columbia $(4 \rightarrow 4)$, Stanford $(8 \rightarrow 8)$, Berkeley $(10 \rightarrow 10)$ keep their positions. Between 2013 [22] and 2017 [30], only 9 universities went out from top100 (IPSA / Karolinska Institutet / Rockefeller / Rutgers / Tsinghua / Amsterdam / Hamburg / Strasbourg / Wrocław) and 9 new universities enter in the top100 (Seoul National University / TU Munich / UC, San Diego / Boulder / Freiburg / Kiel / Marburg / Salamanca / Sydney).

The above numbers and comparisons show that WRWU approach gives a reliable ranking which remains relatively

Table 2. List of the first 10 universities of the 2017 Wikipedia Ranking of World Universities using PageRank algorithm. For a given university, the score $\Theta_{P R}$ is defined by (5), $N_{a}$ is the number of appearances in the top 100 lists of 24 Wikipedia editions, CC is the country code, LC is the language code, and $\mathrm{FC}$ is the foundation century.

\begin{tabular}{lcclccc}
\hline Rank & $\Theta_{P R}$ & $N_{a}$ & University & CC & LC & FC \\
\hline \hline 1st & 2281 & 24 & University of Oxford & UK & EN & 11 \\
2nd & 2278 & 24 & University of Cambridge & UK & EN & 13 \\
3rd & 2277 & 24 & Harvard University & US & EN & 17 \\
4th & 2099 & 24 & Columbia University & US & EN & 18 \\
5th & 1959 & 23 & Yale University & US & EN & 18 \\
6th & 1917 & 24 & University of Chicago & US & EN & 19 \\
7th & 1858 & 23 & Princeton University & US & EN & 18 \\
8th & 1825 & 21 & Stanford University & US & EN & 19 \\
9th & 1804 & 21 & Massachusetts Institute of Technology & US & EN & 19 \\
10th & 1693 & 20 & University of California, Berkeley & US & EN & 19 \\
\hline
\end{tabular}

close to ARWU at different years. At the same time WRWU has about $40 \%$ of different universities compared to ARWU. The origin of this difference is based on variety of cultural views well present in 24 editions. Thus the right panel of Fig. 1 shows a spectacular difference between EN, FR and DE editions: for top 10 universities the German edition has only about $10 \%$ overlap with ARWU while EN and FR have about 50\%. For top 100 this difference still remains significant being approximately $34 \%$ for DE, $43 \%$ for FR and $60 \%$ for EN. Thus the case of German edition demonstrates rather different cultural view on importance of their universities. As discussed in [14] there are clear historical grounds for this difference related to the world dominance of German and Italian universities before 19th century as it is clearly seen in Fig. 10 in [14].

In total for all 24 editions we find 1011 and 1464 different universities with PageRank and CheiRank algorithms respectively. Their geographical distribution over the country world map is shown in Fig. 2. The largest number of top universities is in US but we see a significant numbers also for India, Japan, Germany and France for PageRank which characterizes the university influence. The communicativity is highlighted by CheiRank with top countries being US, India, Japan, France and China. Of course, Hindi, Japan, Chinese editions give certain preference to their own universities but in global the high positions of these universities and countries reflect significant efforts in higher education performed by these countries.

The geographical distributions of top 100 universities of ARWU2017 and WRWU2017 are presented in Fig. 3. For ARWU the top countries are US, UK, Australia and China while for WRWU we find US, Germany, UK and Japan. It is clear that ARWU gives too high significance to Anglo-saxon and Chinese universities while WRWU provides more balanced historical view taking into account a significant role played e.g. by German universities.

A more detailed view on the universities distribution over countries for ARWU and WRWU is shown in Fig. 4. The ranking of WRWU universities inside each country is given in [30].

Table 3. List of the first 10 universities of ARWU2017 [3]. The last columns show the difference between ARWU2017 rank and WRWU2017 rank.

\begin{tabular}{llr}
\hline Rank & ARWU17 & WRWU17 \\
\hline \hline 1st & Harvard University & -2 \\
2nd & Stanford University & -6 \\
3rd & University of Cambridge & +1 \\
4th & Massachusetts Institute of Technology & -5 \\
5th & University of California, Berkeley & -5 \\
6th & Princeton University & -1 \\
7th & University of Oxford & +6 \\
8th & Columbia University & +4 \\
9th & California Institute of Technology & -13 \\
10th & University of Chicago & +4 \\
\hline
\end{tabular}




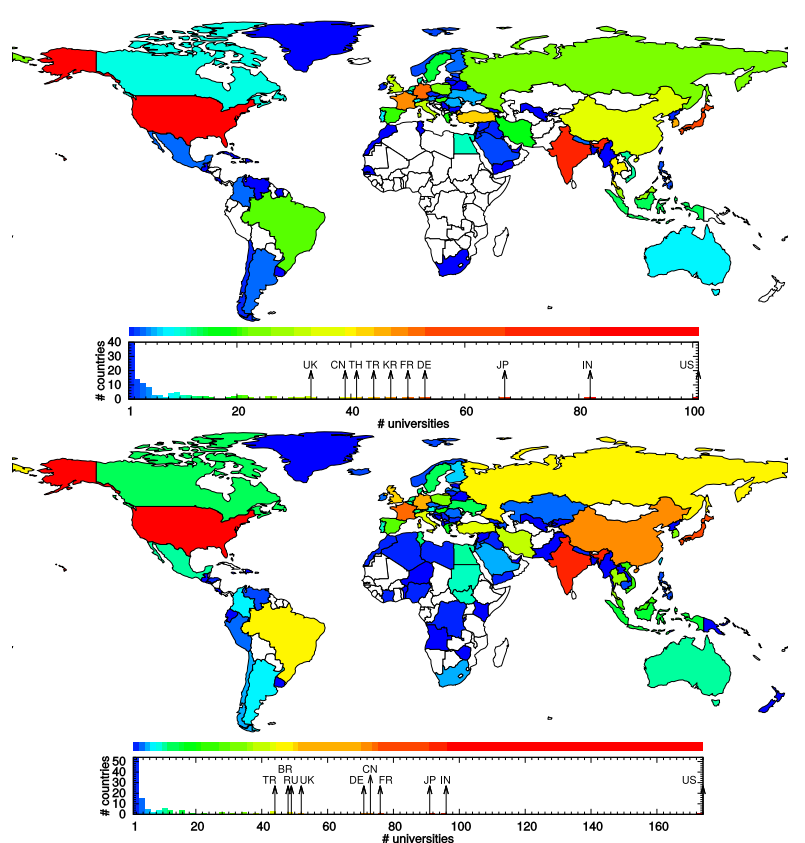

Fig. 2. Geographical distribution of the universities entering the 2017 Wikipedia Ranking of World Universities using PageRank (top panel) and CheiRank algorithms (bottom panel). The total number of universities is 1011 (1464) for WRWU using PageRank (CheiRank) algorithm. US universities are the most numerous: 101 (174) universities for WRWU using PageRank (CheiRank) algorithm. Countries with white color have no universities in the top 100 edition lists. Here and below the color categories are obtained using the Jenks natural breaks classification method [33].

\section{Influence of world universities on countries from English Wikipedia edition}

In this Section we use the REGOMAX approach to analyze the influence of universities on world countries. With this aim the reduced Google matrix is constructed for the subset of articles devoted to the PageRank top 20 universities of ENWRWU (see Tab. 4) and articles devoted to the 85 countries to which belong universities appearing in WRWU (see Tab. 5). Thus the total size of the reduced Google matrix is $n_{r}=105$, to be compared to the global ENWIKI network size which is about 5.4 millions articles.

Table 4. List of the PageRank top 20 universities of English edition WRWU2017. The color code corresponds to the regional location of universities: red for US west coast, orange for US central region, blue for US east coast, and violet for UK.

\begin{tabular}{rl|rl}
\hline Rank & University & Rank & University \\
\hline \hline 1st & Harvard University & 11 th & University of Michigan \\
2nd & University of Oxford & 12 th & Cornell University \\
3rd & University of Cambridge & 13 th & University of California, Los Angeles \\
4th & Columbia University & 14 th & University of Pennsylvania \\
5th & Yale University & 15 th & New York University \\
6th & Stanford University & 16 th & University of Texas at Austin \\
7 th & Massachusetts Intitute of Technology & 17 th & University of Florida \\
8th & University of California, Berkeley & 18 th & University of Edinburgh \\
9th & Princeton University & 19 th & University of Wisconsin-Madison \\
10th & University of Chicago & 20th & University of Southern California \\
\hline
\end{tabular}

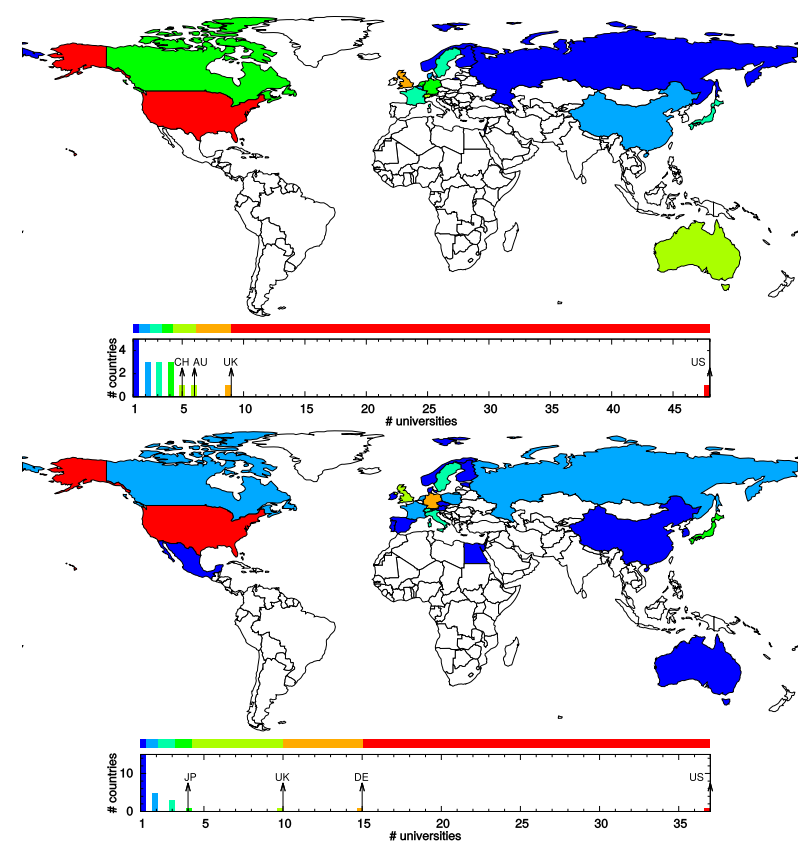

Fig. 3. Geographical distribution of the first 100 universities from ARWU2017 (top panel) and WRWU2017 from PageRank (bottom panel). US universities are the most numerous, 48 universities for ARWU2017 and 37 universities for WRWU2017.
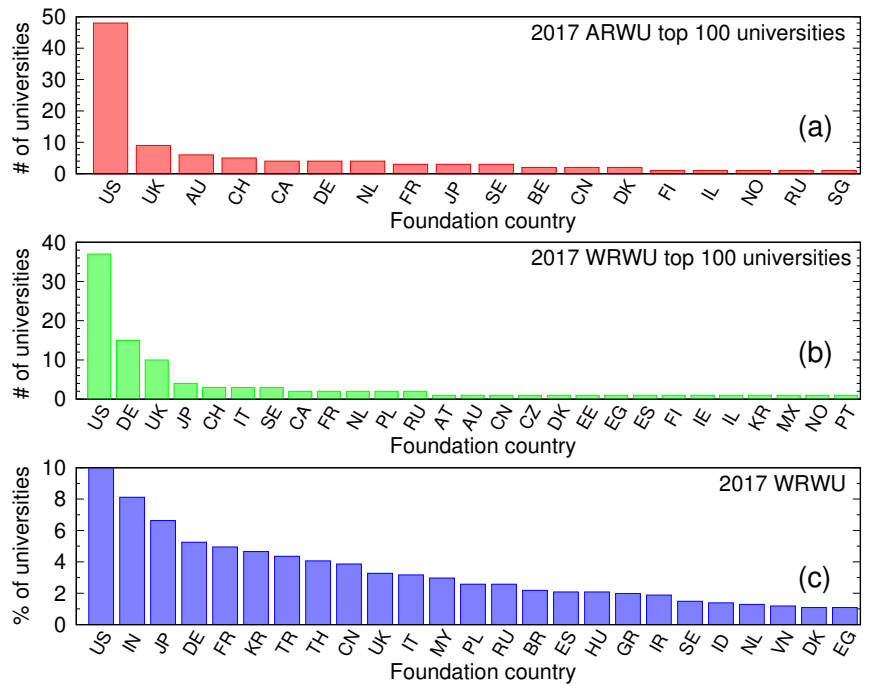

Fig. 4. Distribution over countries of (a) 2017 ARWU top 100 universities and of (b) 2017 WRWU top 100 universities. Panel (c) gives the percentage per country of universities among the 1011 universities listed in 2017 WRWU, countries with less than $10 \%$ are not shown. Countries with equal number of universities are sorted by alphabetic order.

The images of the corresponding reduced Google matrix $G_{\mathrm{R}}$ and its components $G_{\mathrm{rr}}, G_{\mathrm{pr}}$, and $G_{\mathrm{qr}}$, are shown in Fig. 5. As discussed above and in [27] the $G_{\mathrm{pr}}$ component is rather close to the matrix composed by identical columns of PageRank vector of $n_{r}$ nodes, the direct links are presented by the component $G_{\mathrm{rr}}$ and indirect links by $G_{\mathrm{qr}}$ (and related $G_{\mathrm{qrnd}}$ ). The weights of these three components (sum of elements of all columns divided by 
matrix size $n_{r}$ ) are respectively $W_{\mathrm{R}}=1, W_{\mathrm{pr}}=0.948273$, $W_{\mathrm{rr}}=0.0144137$, and $W_{\mathrm{qr}}=0.0373132$. The weights of the components $G_{\mathrm{rr}}$ and $G_{\mathrm{qr}}$ are small, compared to those of $G_{\mathrm{pr}}$, but these two components provide new important information on interactions between nodes. The weight of indirect links is larger than the direct ones $W_{\mathrm{qr}}>W_{\mathrm{rr}}$.

The knowledge of all matrix elements of $G_{\mathrm{R}}$ allows us to determine the influence or sensitivity of a given university $u$ on a given country $c$. To measure the sensitivity we change the matrix element $G_{\mathrm{R}}(u \rightarrow c)$ by a factor $(1+\delta)$ with $\delta \ll 1$, we renormalize to 1 the sum of the column associated to university $u$, and we compute the logarithmic derivative of PageRank probability $P(c)$ associated to country $c: D(u \rightarrow c, c)=d \ln P(c) / d \delta$ (diagonal sensitivity). It is also possible to consider the nondiagonal sensitivity $D\left(u \rightarrow c, c^{\prime}\right)=d \ln P\left(c^{\prime}\right) / d \delta$ when the variation is done for the link from $u$ to $c$ and the derivative of PageRank probability is computed for another country $c^{\prime}$. This approach was already used in $[28,34]$ showing its efficiency.

Table 5. List of the countries associated to the universities appearing in WRWU2017. Countries are ranked from $2017 \mathrm{Wi}$ kipedia English edition using PageRank algorithm.

\begin{tabular}{|c|c|c|c|c|c|}
\hline Rank & University & $\mathrm{CC}$ & Rank & University & $\mathrm{CC}$ \\
\hline  & United States & US & $\overline{\overline{444}}$ & Chile & 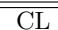 \\
\hline 2 & France & FR & 45 & Republic of Ireland & IE \\
\hline 3 & Germany & $\mathrm{DE}$ & 46 & Singapore & SG \\
\hline 4 & United Kingdom & $\mathrm{UK}$ & 47 & Serbia & RS \\
\hline 5 & Iran & IR & 48 & Vietnam & $\mathrm{VN}$ \\
\hline 6 & India & IN & 49 & Nepal & NP \\
\hline 7 & Canada & $\mathrm{CA}$ & 50 & Estonia & $\mathrm{EE}$ \\
\hline 8 & Australia & $\mathrm{AU}$ & 51 & Iraq & $\mathrm{IQ}$ \\
\hline 9 & China & $\mathrm{CN}$ & 52 & Bangladesh & $\mathrm{BD}$ \\
\hline 10 & Italy & IT & 53 & Syria & SY \\
\hline 11 & Japan & JP & 54 & Myanmar & MM \\
\hline 12 & Russia & RU & 55 & Slovakia & SK \\
\hline 13 & Brazil & $\mathrm{BR}$ & 56 & Venezuela & $\mathrm{VE}$ \\
\hline 14 & Spain & ES & 57 & Morocco & MA \\
\hline 15 & Netherlands & NL & 58 & Cuba & $\mathrm{CU}$ \\
\hline 16 & Poland & $\mathrm{PL}$ & 59 & Puerto Rico & PR \\
\hline 17 & Sweden & $\mathrm{SE}$ & 60 & Saudi Arabia & SA \\
\hline 18 & Mexico & MX & 61 & Lithuania & LT \\
\hline 19 & Turkey & TR & 62 & Lebanon & LB \\
\hline 20 & Romania & $\mathrm{RO}$ & 63 & Cyprus & $\mathrm{CY}$ \\
\hline 21 & South Africa & $\mathrm{ZA}$ & 64 & Latvia & LV \\
\hline 22 & Norway & $\mathrm{NO}$ & 65 & Belarus & BY \\
\hline 23 & Switzerland & $\mathrm{CH}$ & 66 & United Arab Emirates & $\mathrm{AE}$ \\
\hline 24 & Philippines & $\mathrm{PH}$ & 67 & Uruguay & UY \\
\hline 25 & Austria & AT & 68 & North Korea & $\mathrm{KP}$ \\
\hline 26 & Belgium & $\mathrm{BE}$ & 69 & Yemen & YE \\
\hline 27 & Argentina & $\mathrm{AR}$ & 70 & Costa Rica & CR \\
\hline 28 & Indonesia & ID & 71 & Tunisia & $\mathrm{TN}$ \\
\hline 29 & Greece & GR & 72 & Jordan & JO \\
\hline 30 & Denmark & DK & 73 & Guatemala & GT \\
\hline 31 & South Korea & $\mathrm{KR}$ & 74 & Greenland & GL \\
\hline 32 & Israel & IL & 75 & Dominican Republic & DO \\
\hline 33 & Hungary & $\mathrm{HU}$ & 76 & Uzbekistan & $\mathrm{UZ}$ \\
\hline 34 & Finland & FI & 77 & Kuwait & KW \\
\hline 35 & Egypt & EG & 78 & Qatar & QA \\
\hline 36 & Portugal & $\mathrm{PT}$ & 79 & Senegal & SN \\
\hline 37 & Taiwan & TW & 80 & El Salvador & SV \\
\hline 38 & Ukraine & UA & 81 & Suriname & SR \\
\hline 39 & Czech Republic & $\mathrm{CZ}$ & 82 & Faroe Islands & $\mathrm{FO}$ \\
\hline 40 & Malaysia & MY & 83 & Brunei & $\mathrm{BN}$ \\
\hline 41 & Thailand & $\mathrm{TH}$ & 84 & Palestine & PS \\
\hline 42 & Colombia & $\mathrm{CO}$ & 85 & Georgia & $\mathrm{GE}$ \\
\hline 43 & Bulgaria & BG & & & \\
\hline
\end{tabular}
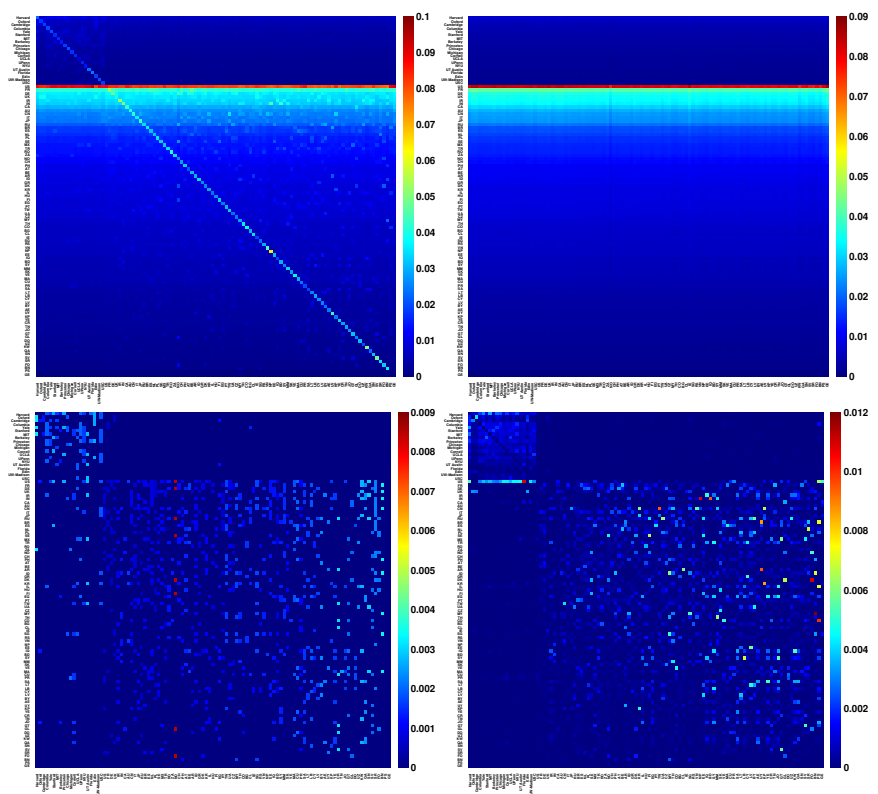

Fig. 5. Reduced Google matrix $G_{\mathrm{R}}$ for the first 20 universities ranked in ENWRWU (Tab. 4) and the 85 countries to which universities from WRWU belong (Tab. 5). The full reduced Google matrix $G_{\mathrm{R}}$ is presented in top left panel, $G_{\mathrm{pr}}$ in top right panel, $G_{\mathrm{rr}}$ in bottom left panel, and $G_{\mathrm{qrnd}}$ in bottom right panel. The weights of $G_{\mathrm{R}}$ matrix components are $W_{\mathrm{R}}=1$, $W_{\mathrm{pr}}=0.948273, W_{\mathrm{rr}}=0.0144137$, and $W_{\mathrm{qr}}=0.0373132$. The node order presents first 20 universities in order of Tab. 4 and then 85 countries in order of Tab. 5 .

The world maps of university influence on countries, expressed by the diagonal sensitivity $D(u \rightarrow c, c)$ for 4 selected universities, are shown in Fig. 6.

For Harvard the most sensitive country is South Africa (ZA) due to the well known scandal linked to Harvard investments in apartheid ZA pointed on the Harvard wikipage. Puerto Rico also appears on this wikipage in relation with oldest universities in the America. However, next influenced countries are Georgia (GE), Israel (IL) and Ireland (IR) which are not present on the wikipage which appearance we attribute to indirect links.

For Chicago the most influenced countries are Singapore (SG), Puerto Rico (PR) and India (IN). The first two countries SG, PR are not present on the wikipage showing that indirect links play an important role for them. India has a direct link related to the following facts: Chicago campus opened in India and a faculty member was erstwhile governor of India central bank.

For Stanford the top countries are Spain (ES), present on the wikipage, $\mathrm{PR}$ and $\mathrm{ZA}$ appearing due to indirect links.

For Oxford the top three countries are Jordan (JO), appearing of wikipage since Abdullah II of JO has been educated at Oxford; Iraq (IQ), also appearing on wikipage since T. E. Lawrence educated at Oxford played a major role in establishing and administering the modern state of IQ; IR which is not present on wikipage but has close links with UK. 


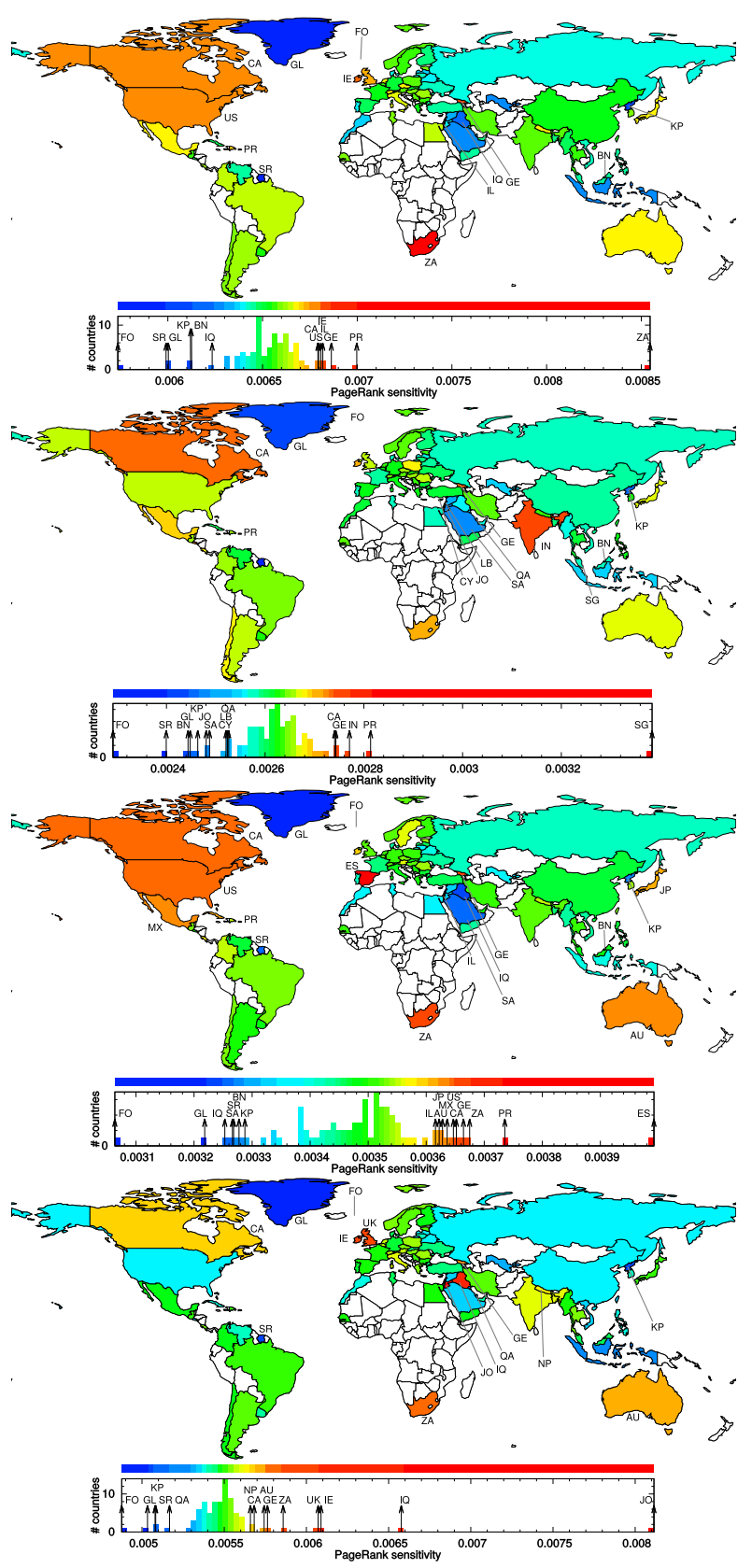

Fig. 6. World map of diagonal sensitivity of world countries $D(u \rightarrow c, c)$ to the change of the reduced Google matrix link university $u \rightarrow$ country $c$. The cases of 4 universities are shown from top to bottom: Harvard University, University of Chicago, Stanford University, and University of Oxford.

Examples of nondiagonal sensitivity are shown in Fig. 7 for the link variation Harvard University to US and University of Oxford to UK. For the link Harvard $\rightarrow$ US the most influenced countries are Suriname (SR), People's Republic of Korea (KP) and Puerto Rico (PR). For the link variation Oxford $\rightarrow \mathrm{UK}$ the most influenced countries are Qatar (QA), Ireland (IR) and Singapore (SG). By definition the nondiagonal sensitivity contains indirect effects and it is not so easy to find the pathways of links which are

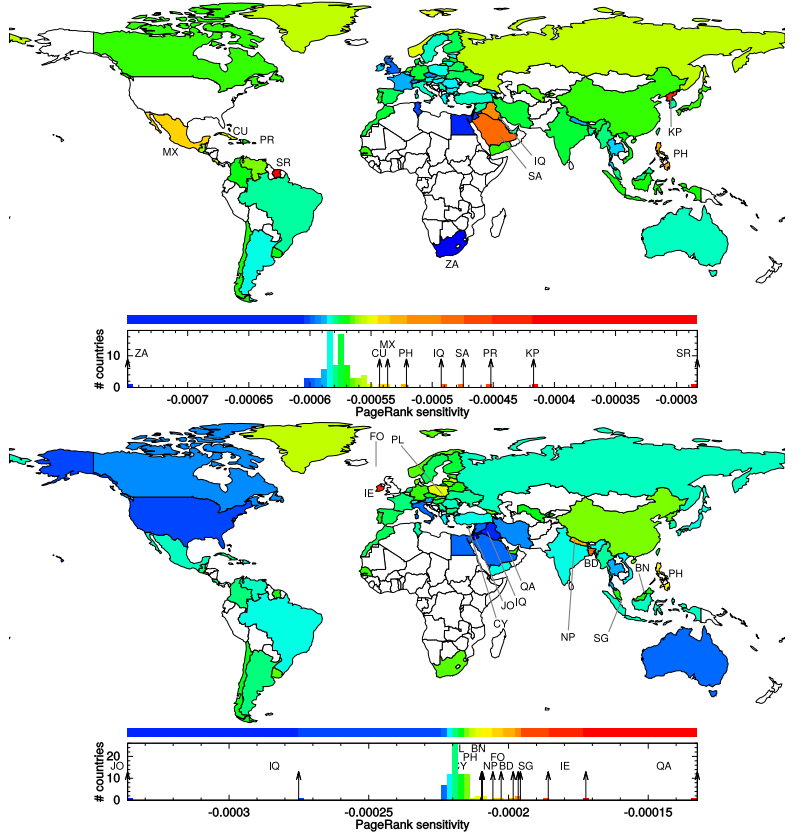

Fig. 7. World map of nondiagonal sensitivity $D\left(u \rightarrow c, c^{\prime}\right)$ of country $c^{\prime}$ to the change of the reduced Google matrix link Harvard $\rightarrow$ US (top panel) and Oxford $\rightarrow$ UK (bottom panel). Red (blue) color corresponds to the greatest (lowest) absolute value. Diagonal values $D\left(u \rightarrow c, c^{\prime}=c\right)$ are not shown.

responsible for this dominant influence. These examples show the strength of reduced Google matrix approach.

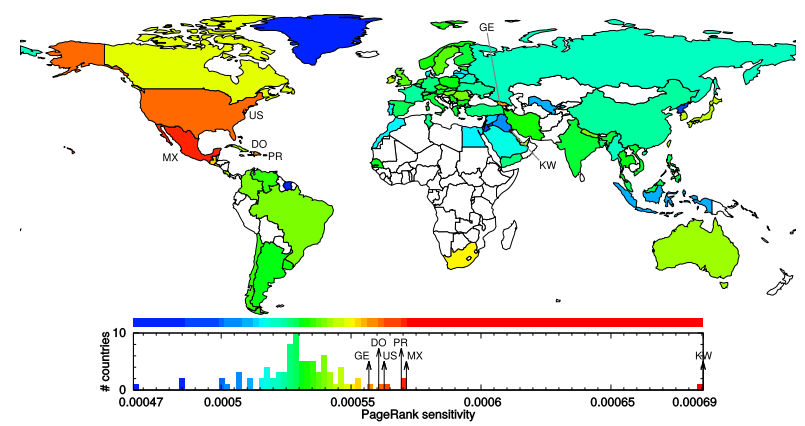

Fig. 8. Same as in Fig. 6 with diagonal sensitivity $D$ ("Rice University" $\rightarrow c, c$ ) to the change of the reduced Google matrix link Rice University $\rightarrow$ country $c$.

Finally we discuss an example of Rice University. It has rank 74 in ARWU2017 being at position 37 inside USA, but according to WRWU2017 its PageRank position is only 357 and 56 inside USA. This shows that the Wikipedia article of Rice University is not sufficiently developed and its visibility via Wikipedia is rather low and can be improved by more skillful organization of its wikipage. We note that Wikipedia visibility of a university plays rather important role since very often it is on top lines of Google search and many language editions spread the wikipage content world wide free of charge. Also the WRWU position 357 is only due to appearance of Rice in top 100 


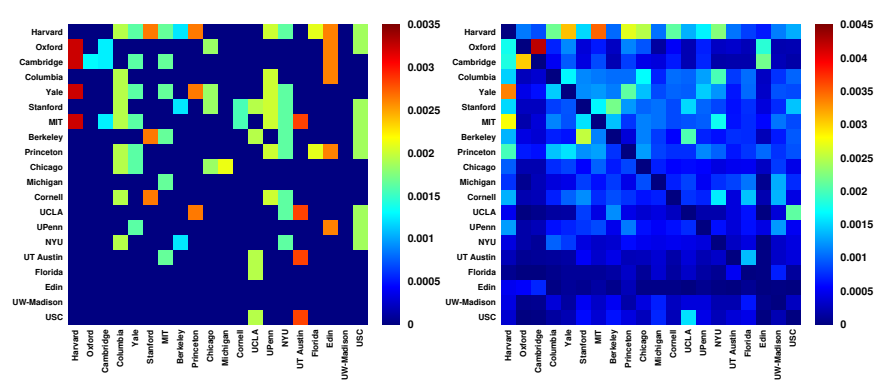

Fig. 9. Matrices $G_{\mathrm{rr}}$ (left panel) and $G_{\mathrm{qrnd}}$ (right panel) for top20 ENWRWU (Tab. 4). The universities are ordered by their PageRank index as in Tab. 4. The matrix weights are $W_{\text {rr }}=0.00877$ and $W_{\text {qrnd }}=0.01381$. Color shows the strength of matrix elements. The same components for top 20 universities of FRWIKI, DEWIKI, RUWIKI are available in Figs. SI3, SI4, SI5.

universities of $\mathrm{FA}, \mathrm{KO}$, and $\mathrm{TH}$ language editions probably due to wikipage creation and information given by Rice alumni speaking these languages. This indicates an importance of links between university and its alumni.

The world influence of Rice University, expressed via its diagonal sensitivity, is shown in Fig. 8. The most influenced countries are Kuwait (KW), Mexico (MX) and Puerto Rico (PR). These countries are not present on the wikipage of Rice University and their appearance is related to indirect links.

\section{Reduced networks of world universities}

In this Section we analyze the interactions between top 20 universities obtained from the REGOMAX approach. We consider the different cultural views from EN, FR, DE, RU editions and make a comparative analysis. For EN edition we also consider the links of selected universities with countries.

\subsection{Top 20 universities in English Wikipedia edition}

Top 20 PageRank universities of ENWRWU2017 are given in Tab. 4. They are either US or UK universities. We define 4 regional groups with their PageRank leaders: Stanford University for US west coast, University of Chicago for US central region, Harvard University for US east coast, and University of Oxford for UK; each group is marked by color in Tab. 4.

The components of reduced Google matrix describing direct links $G_{\mathrm{rr}}$ and indirect links $G_{\text {qrnd }}$ are shown in Fig. 9 (only nondiagonal links are shown for $G_{\mathrm{qr}}$ ). The weight of indirect nondiagonal links is about $50 \%$ percent stronger than the weight of direct links. This shows the importance of indirect interactions between top 20 universities.

To characterize the importance of direct and indirect links we consider the sum of two matrix components given by $G_{\mathrm{rr}}+G_{\mathrm{qrnd}}$. From this matrix we construct the network of friends of regional leaders shown in Fig. 10 (the

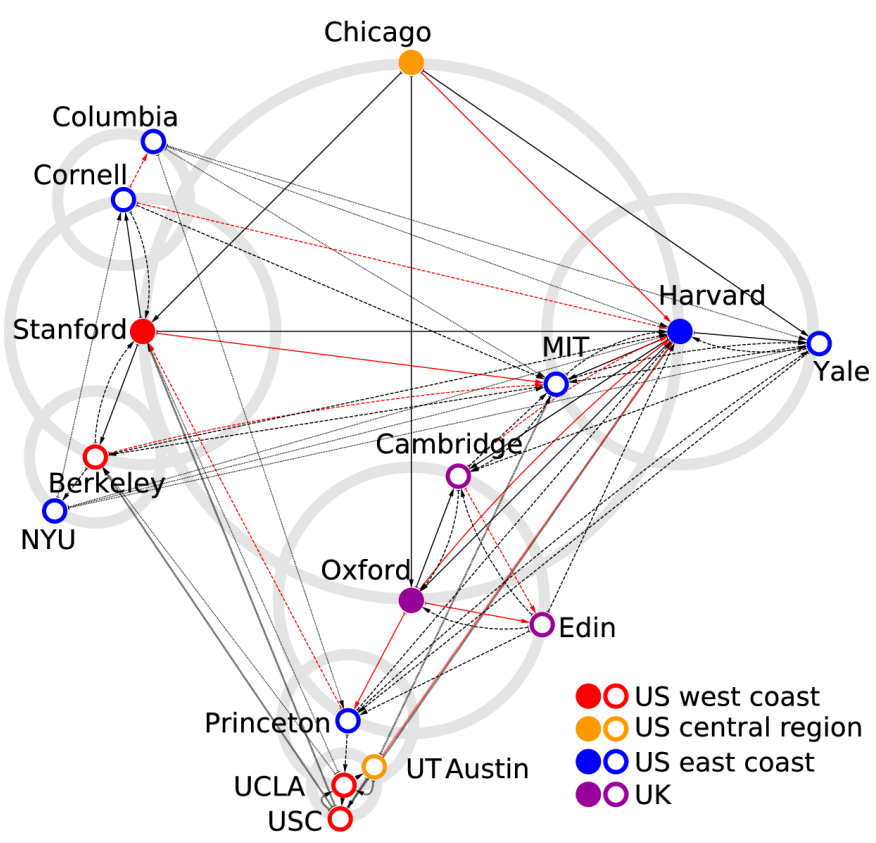

Fig. 10. Network of friends of top 20 PageRank universities of ENWRWU obtained from matrix of direct and indirect links $G_{\mathrm{rr}}+G_{\mathrm{qrnd}}$. Color filled nodes are regional leaders. Red links are purely hidden links, i.e., no corresponding adjacency matrix entry. We obtain 4 friendship levels (gray circles). Links originating from 1st level universities are presented by solid lines, from 2 nd level by dashed lines, from 3rd level by doted lines, and from 4th level by "Y" symbol lines.

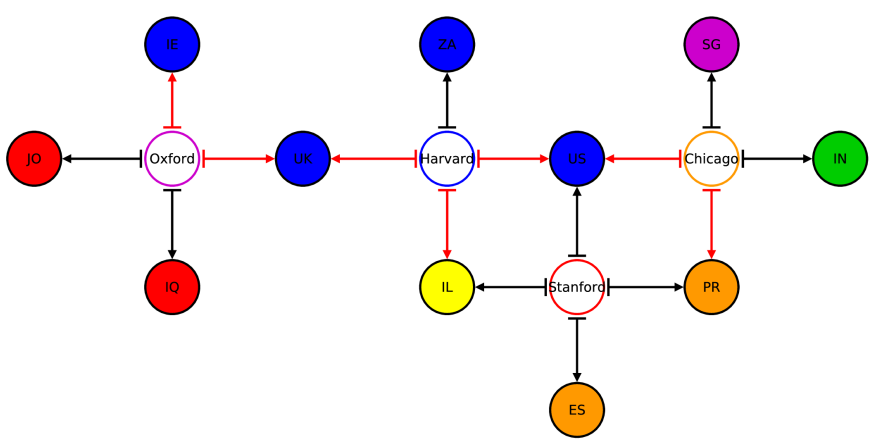

Fig. 11. Network of friends from $G_{\mathrm{rr}}+G_{\text {qrnd }}$ associated to the top20 ENWRWU and 85 countries listed in Tab. 5. For each regional leaders, Stanford University, University of Chicago, Harvard University, University of Oxford, the four strongest links to one of the 85 countries listed in Tab. 5 are presented. Universities (countries) are represented by empty (full) nodes. The color code for countries depends on the main spoken language: blue for English, red for Arabic, orange for Spanish, violet for Chinese, green for Hindi, and yellow for Hebrew. Red links are purely indirect links and black ones are from direct links.

drawings of networks have been produced using Cytoscape [35]). First we place the regional leader on a circle (1st level of possible friendship). From a regional leader we look at the four biggest outgoing links in $G_{\mathrm{rr}}+G_{\mathrm{qrnd}}$, these four links define the four best friends of the regional leader. If these friends are not present in the network of 
friends, i.e., they are not themselves regional leaders, then they are placed on the circle around the regional leader (2nd level of friendship). If several regional leaders share the same friend, by preference, the friend is placed in the circle around the leader of its region. Then, from each friends of regional leaders, we define in the same way four new friends. Each new friend is either already placed in the friendship network or not. If the new friend is not present, it is placed in the circle around the corresponding friend of regional leader (3rd level of friendship). If a new friend is shared by several friends of regional leaders, the new friend is placed by preference on the circle around the friend of regional leader belonging to its region. In the same manner we then define the 4 th level of friendship and so on. The procedure continues until no new friends can be placed on the friendship network (because already placed on it). For the 2017 ENWRWU top20 the procedure stops after four levels of friendship. A red arrow represents a pure hidden link, i.e., a link from university $u$ to university $u^{\prime}$ with a null adjacency matrix entry, $A_{u^{\prime} u}=0$, or otherwise stated, with a minimal value in $G_{\mathrm{rr}},\left(G_{\mathrm{rr}}\right)_{u^{\prime} u}=(1-\alpha) / N$.

This network presentation of friends in Fig. 10 shows that the close friends are mainly located in same region; thus MIT, Harvard, Yale form one group of east coast, Oxford, Cambridge, Edinburgh are friends inside UK. However, there are also inter-regional friends formed by Princeton, UCLA, USC, UT Austin and proximity between Stanford, Berkeley and Cornell. The direct links shown in black play an important role but indirect links shown in red are also present and significant like e.g. MIT being indirect friend of Stanford, Harvard being indirect friend of Chicago and Oxford, and Edinburgh, Princeton being indirect friend of Oxford.

We also consider countries which are friends of each regional university leader as shown in Fig. 11. For this we consider the matrix elements of $G_{\mathrm{rr}}+G_{\text {qrnd }}$ constructed for top 20 universities of Tab. 4 and 85 countries listed in Tab. 5. For each regional leader university we select top 4 friendliest countries. The network of country-university friends is presented in Fig. 11 with countries marked by colors corresponding to mostly spoken language. Thus we see that countries friends of Oxford are mostly Arab and English speaking countries; countries friends of Harvard are dominantly from English speaking countries excepting one Hebrew speaking country; friends of Stanford are two Spanish speaking countries, one English and one He-

Table 6. List of top 20 PageRank universities of French edition WRWU2017. The color code corresponds to the country location of universities: blue for US, violet for UK, red for FR, green for $\mathrm{CA}$, and yellow for $\mathrm{BE}$.

\begin{tabular}{rl|rl}
\hline Rank & University & Rank & University \\
\hline \hline 1st & Harvard University & 11 th & University Laval \\
2nd & University of Oxford & 12 th & Pantheon-Sorbonne University \\
3rd & École polytechnique & 13 th & Princeton University \\
4th & University of Cambridge & 14 th & University of California, Berkeley \\
5th & École normale supérieure & 15 th & Paris-Sorbonne University \\
6th & Massachusetts Institute of Technology & 16 th & Université libre de Bruxelles \\
7th & Yale University & 17 th & University of Montreal \\
8th & Columbia University & 18 th & Université catholique de Louvain \\
9 9th & Stanford University & 19 th & Paris Nanterre University \\
10th & École pratique des hautes études & 20 th & University of Chicago \\
\hline
\end{tabular}

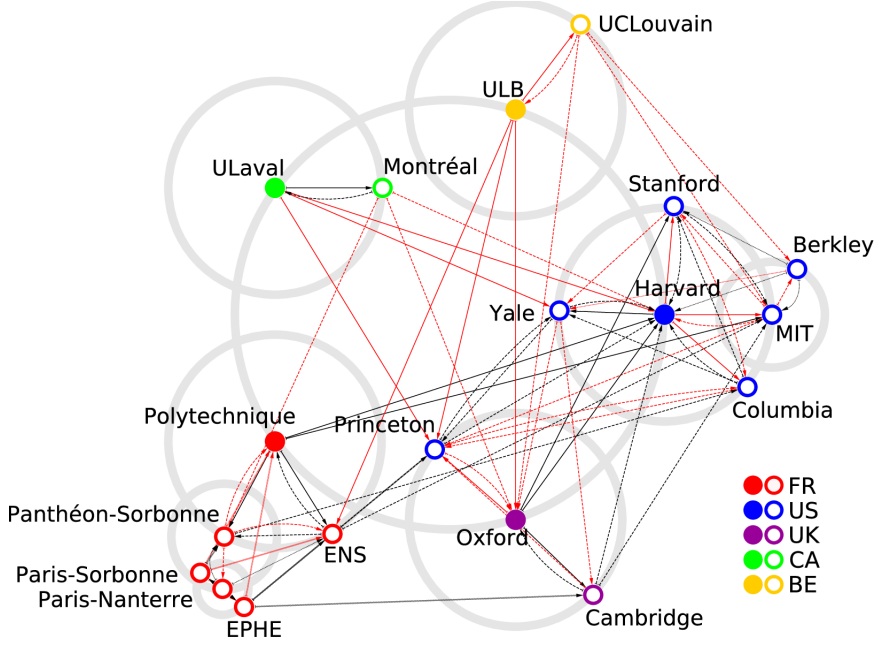

Fig. 12. Same as in Fig. 10 for PageRank top 20 universities of FRWIKI2017 from Tab. 6. Color filled nodes are country leaders. Red links are purely hidden links, i.e., no corresponding adjacency matrix entry. We obtain 4 friendship levels (gray circles). Links originating from 1st level universities are presented by solid lines, from 2nd level by dashed lines, from 3rd level by doted lines, and from 4 th level by "\" symbol lines.

brew; Chicago is mostly diversified having English, Hindi, Chinese and Spanish speaking friends.

\subsection{Top 20 universities in French Wikipedia edition}

Here we consider the cultural view of FRWIKI on top 20 universities and their interactions. We select from FRWIKI2017 top PageRank universities listed in Tab. 6. These universities belong to 5 countries (US, UK, FR, CA, BE) marked by corresponding color. Top PageRank university of each country is considered as a country leader. Similar to the case of Fig. 10 we obtain from the reduced Google matrix components $G_{\mathrm{rr}}+G_{\mathrm{qrnd}}$ of these 20 universities the network of friends shown in Fig. 12.

The obtained network of friends of Fig. 12 shows clear cluster of universities inside their own countries. However, the inter-country links are well present and they are mainly indirect links (shown in red) pointing toward English speaking universities. Thus Princeton is indirect friend of Oxford, ULaval and ULB; Yale and Harvard are indirect friends of ULaval. Since we consider FRWIKI there are 6 French universities being the next in number after US with 7 universities among top 20. However, US universities are strongly linked with universities of Canada, Belgium and UK while French universities are weakly linked to other countries. This FRWIKI-analysis demonstrate certain world isolation of French universities. Also from FRWIKI point of view, the leading English speaking universities in Fig. 12 form an invariant subset from which a random surfer cannot escape: the friends of these universities are uniquely English speaking universities. 


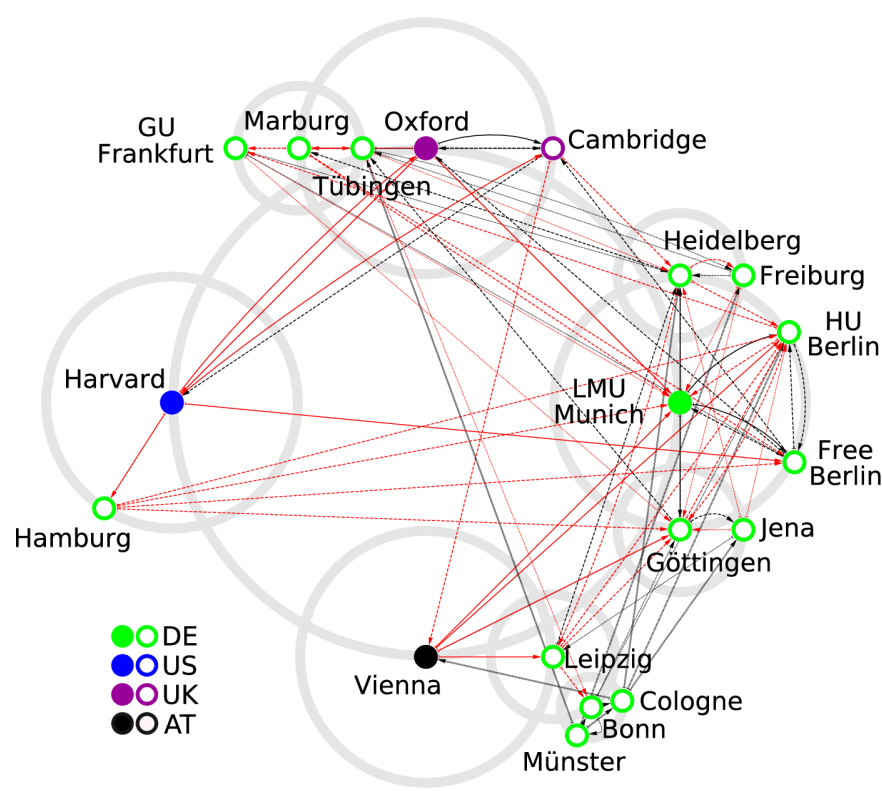

Fig. 13. Same as in Fig. 10 for PageRank top 20 universities of DEWIKI2017 from Tab. 7. Color filled nodes are country leaders. Red links are purely hidden links, i.e., no corresponding adjacency matrix entry. We obtain 4 friendship levels (gray circles). Links originating from 1st level universities are presented by solid lines, from 2 nd level by dashed lines, from 3rd level by doted lines, and from 4 th level by "\" symbol lines.

\subsection{Top 20 universities in German Wikipedia edition}

Top 20 PageRank universities are given in Tab. 7. The network of friends, constructed for these 20 nodes from the matrix components $G_{\mathrm{rr}}+G_{\mathrm{qrnd}}$ of the reduce Google matrix, is shown in Fig. 13. A specific point of these top 20 universities is that there are only 3 non-German-speaking universities (Harvard, Oxford, Cambridge). This is due to the already discussed feature of DEWIKI which gives strong preference to German universities (see right panel of Fig. 1). The universities belong only to 4 countries AT, DE, UK, US. The main cluster are formed around LMU Munich and Vienna however GU Frankfurt, Marburg and Tubingen are closely linked with Oxford and Cambridge; Hamburg is linked with Harvard. It should be pointed that there is a dominance of indirect links which are also linking different countries.

Table 7. List of top 20 PageRank universities of German edition WRWU2017. The color code corresponds to the country location of universities: green for DE, blue for US, violet for UK, and black for AT.

\begin{tabular}{rl|rl}
\hline Rank & University & Rank & University \\
\hline \hline 1st & Ludwig Maximilian University of Munich & 11 th & University of Freiburg \\
2nd & Humboldt University of Berlin & 12 th & University of Cologne \\
3rd & University of Göttingen & 13 th & University of Muinster \\
4th & Heidelerg University & 14 h & University of Oxford \\
5th & Free University of Berlin & 15 th & University of Hamburg \\
6th & University of Vienna & 16 th & Goethe University Frankfurt \\
7 th & University of Tübingen & 17 th & University of Cambridge \\
8th & Harvard University & 18 th & University of Marburg \\
9 th & University of Bonn & 19 hh & University of Kiel \\
10th & Leipzig University & 20 th & University of Jena \\
\hline
\end{tabular}

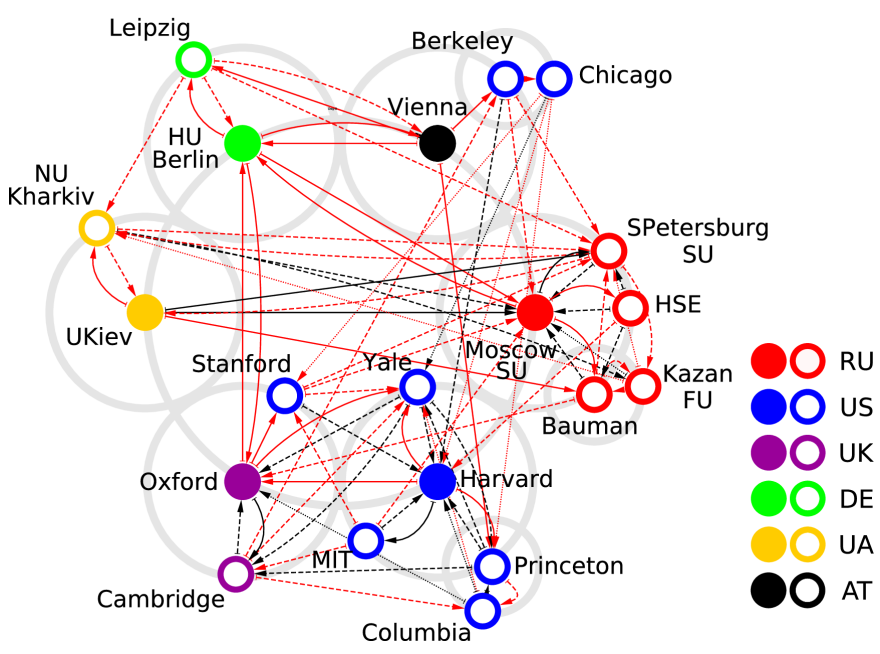

Fig. 14. Same as in Fig. 10 for PageRank top 20 universities of RUWIKI2017 from Tab. 8. Color filled nodes are country leaders. Reduced network from top20 RUWRWU $G_{\mathrm{rr}}+G_{\mathrm{qrnd}}$. Color filled nodes are regional leaders. Red links are purely hidden links, i.e., no corresponding adjacency matrix entry. We obtain 3 acquaintance levels (gray circles). Links originating from 1st level universities are presented by solid lines, from 2nd level by dashed lines, and from 3rd level by doted lines.

\subsection{Top 20 universities in Russian Wikipedia edition}

Top 20 PageRank universities are given in Tab. 8. The network of friends, constructed for these 20 nodes from the matrix components $G_{\mathrm{rr}}+G_{\mathrm{qrnd}}$ of the reduce Google matrix, is shown in Fig. 14. Among these 20 universities there 8 from US, 5 from Russia, 2 from Ukraine, 2 from Germany (its former DDR part), 2 from UK and 1 from Austria so that there are 6 different countries. The clusters of universities are mainly linked with their own countries even if there is very close proximity between UK and US even if Berkeley and Chicago are in the circle proximity of Vienna. The main intercountry links are mainly indirect (except direct links between Kiev pointing to Moscow and St. Petersburg which were all inside former USSR). It is interesting to note that German university, belonging to the former DDR part of Germany, have strong links with Russian universities, showing that the links inside Soviet block are still significant even if Wikipedia had been created well after disappearance of DDR.

Table 8. List of top 20 PageRank universities of German edition WRWU2017. The color code corresponds to the country location of universities: red for RU, blue for US, violet for UK, green for DE, black for AT, and yellow for UA.

\begin{tabular}{rl|rl}
\hline Rank & University & Rank & University \\
\hline \hline 1st & Moscow State University & 11 h & Kazan Federal University \\
2nd & Saint Petersburg State University & 12 th & National University of Kharkiv \\
3rd & Harvard University & 13 th & Stanford University \\
4th & University of Oxford & 14th & Princeton University \\
5th & University of Cambridge & 15th & University of Chicago \\
6th & Massachusets Institute of Technology & 16 th & Higher School of Economics \\
7th & Yale University & 1th & Bauman Moscow State Technical University \\
8th & Columbia University & 18 th & Leipzig University \\
9th & Kyiv University & 19th & University of Vienna \\
10th & Humboldt University of Berlin & 20th & University of California, Berkeley \\
\hline
\end{tabular}



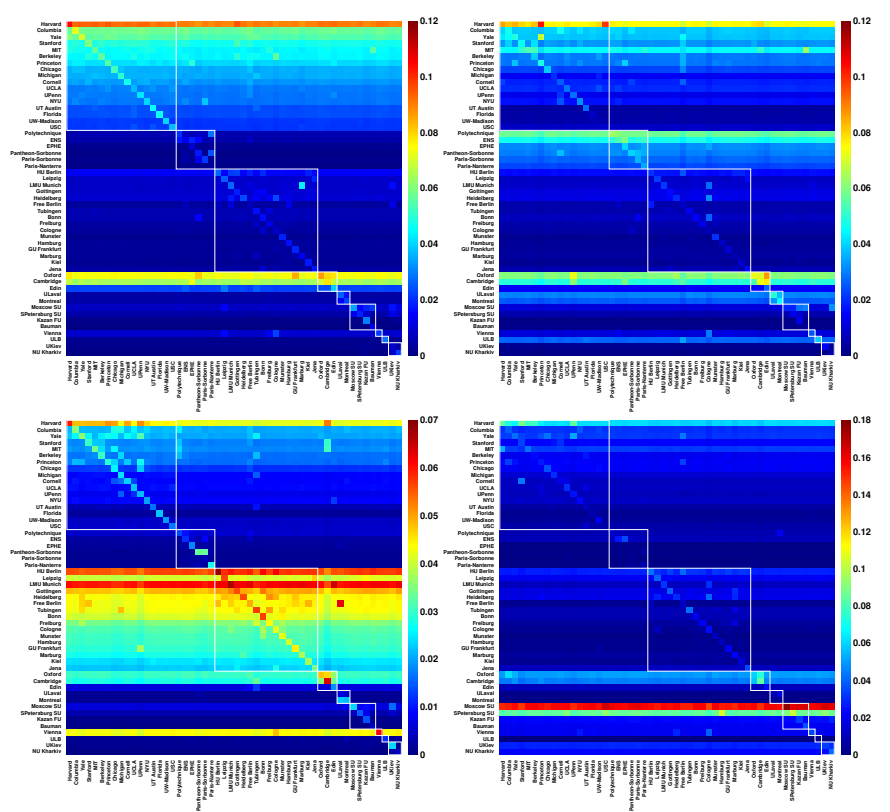

Fig. 15. Reduced Google matrix $G_{\mathrm{R}}$ for universities listed in Tab. 9 computed from EN (top left), FR (top right), DE (bottom left), and RU (bottom right) Wikipedia editions; for each edition nodes have the same order as in Tab. 9. The images of components $G_{\mathrm{pr}}, G_{\mathrm{rr}}, G_{\mathrm{qrnd}}$ are given in Figs. SI6, SI7, SI8.

\subsection{Comparison between English, French, German, and Russian Wikipedia editions}

In this subsection we perform a comparison of different cultural views of DEWIKI, ENWIKI, FRWIKI and RUWIKI on top universities. With this aim we take 20 PageRanked universities of each of these editions. This gives us 52 different universities presented in these editions. The list of them is given in Tab. 9. Each of these universities is attributed to its own foundation country shown by colors in this Table. There are 9 different countries: US, FR, DE, $\mathrm{UK}, \mathrm{CA}, \mathrm{RU}, \mathrm{AT}, \mathrm{BE}$ and UA.

Then we perform the reduced Google matrix analysis for these 52 universities for each edition constructing $G_{\mathrm{R}}$ and its 3 components. The matrices $G_{\mathrm{R}}$ for each edition are shown in Fig. 15 . The $G_{\mathrm{pr}}, G_{\mathrm{rr}}, G_{\mathrm{qrnd}}$ matrix components are available in Figs. SI6, SI7, SI8 with their weights which are similar to those given in Fig. 5; the weights of direct and indirect links are comparable. From Fig. 15 we see that each edition has its own view on these 52 universities. Indeed, there is a clear tendency that edition rank higher universities belonging to the countries with edition language, e.g. RUWIKI places Moscow and St. Petersburg universities on top PageRank positions with a similar situation for DEWIKI. Using the matrix components of $G_{\mathrm{rr}}+G_{\mathrm{qrnd}}$ we analyze the network of friend of 52 universities from the view point of ENWIKI, FRWIKI, DEWIKI and RUWIKI. The approach is the same as those used in network of friends discussed in the previous subsection.

The network of top friends for 52 universities in ENWIKI is shown in Fig. 16. We see that the majority of links

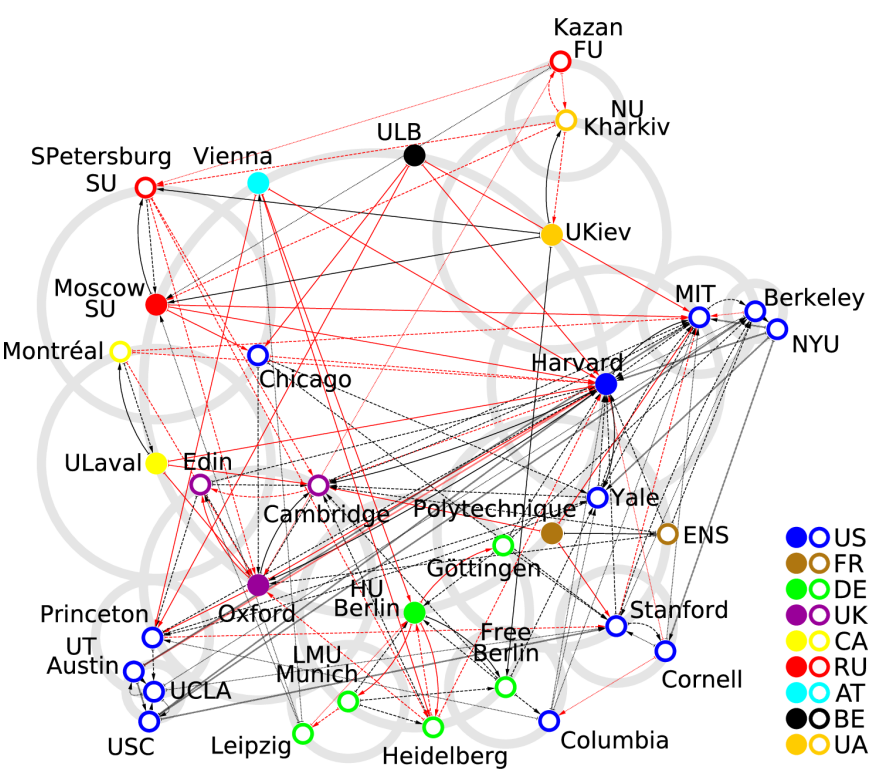

Fig. 16. Network of friends of 52 universities listed in Tab. 9 computed from $G_{\mathrm{rr}}+G_{\mathrm{qrnd}}$ of ENWIKI. Color filled nodes are country leaders (same colors as in the Tab. 9). Red links are purely hidden links, i.e., no corresponding adjacency matrix entry. We obtain 4 acquaintance levels (gray circles). Links originating from 1st level universities are presented by solid lines, from 2 nd level by dashed lines, from 3rd level by doted lines, and from 4 th level by " " symbol lines.

are indirect (red) comparing to the direct links (black). As expected two clusters of English speaking universities are well visible; in fact as in Fig. 12, these English speaking universities form an invariant subspace from which a random surfer cannot escape. These universities act as an attractor subset in this friendship network. Chicago is located aside as it was already visible in previous subsection

Table 9. List of the 52 different universities appearing in the top20s of the EN, FR, DE, RU WRWU. Color code corresponds to country: US, FR, DE, UK, CA, RU, AT, BE and UA. Universities are ordered by countries (country groups are ordered according to PageRanking of 2017 English Wikipedia). Within country groups universities are ordered according to PageRanking of 2017 English Wikipedia.

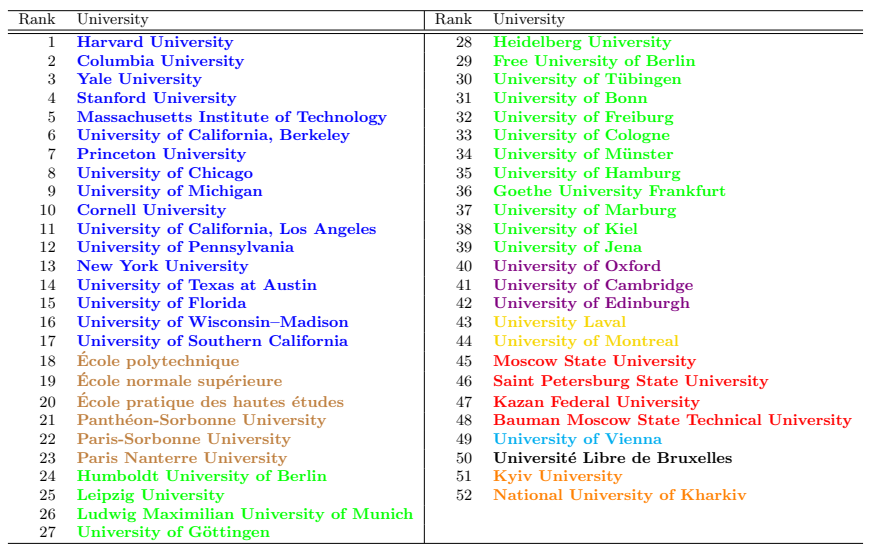




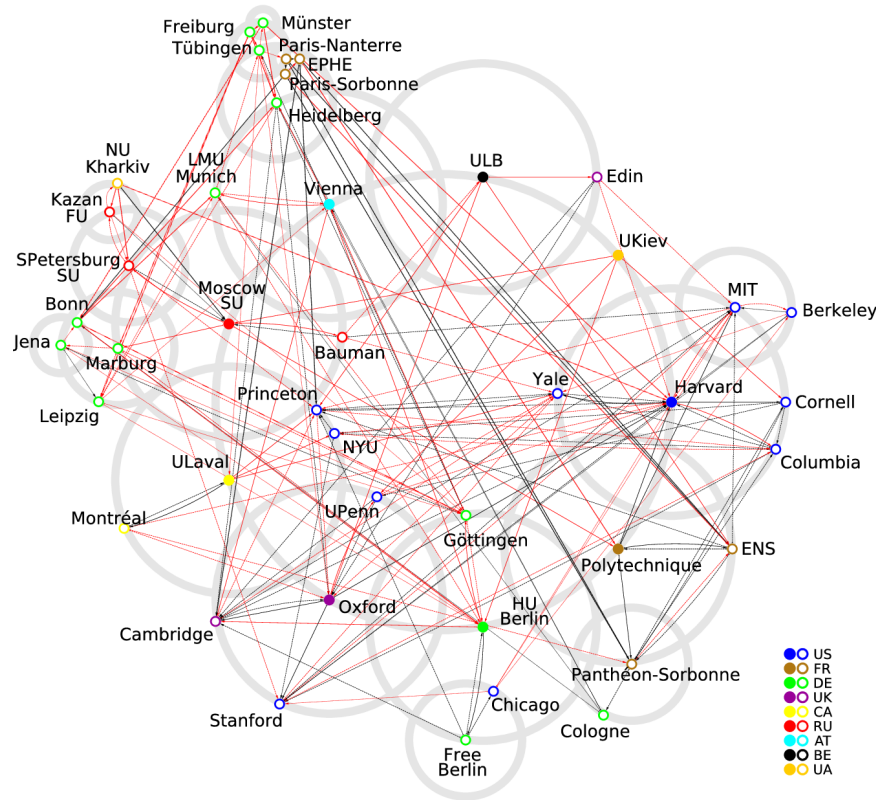

Fig. 17. Same as in Fig. 16 but from FRWIKI.

(see Fig. 10). A compact cluster of German universities is also well visible. We point that there are only 2 isolated French universities among top friends appearing in Fig. 16; no university from other countries points toward these 2 universities, and these 2 universities point exclusively toward UK/US universities. In total this network of top friends has 13 US universities, 6 of DE, 3 of UK, 3 of RU, 2 of UA, 2 of CA, 2 of FR. Since the network is obtained from ENWIKI it is understandable that US universities (with UK ones) form the majority. However, German universities show their strength and significant influence. Comparing to them French university group is small and not significant being placed behind Russian universities. This network clearly shows the weak representation and influence of French universities that reflects a certain reality.

The network of top friends from FRWIKI is shown in Fig. 17. Here we have the dominance of 13 German universities, followed by 11 of US, 6 of France and 4 of Russia. Still the indirect links play a dominant or comparable role with the direct links. In this network the cluster structure is less visible, however as in Figs. 12 and 16 the cluster of US-UK universities is central and acts as an attractor subset.

Fig. 18 shows the network of top friends from DEWIKI. Here, naturally, the dominance of 14 German universities remains, to be compared with 11 of US, 3 of France, 3 of UK and 2 of Russia. In global DE universities are distributed over 3 clusters, and US over 2 clusters.

The network of top friends from RUWIKI is shown in Fig. 19. The dominance of German universities is well present here with 16 of them followed by 10 of US, 4 of Russia and 3 of UK. Three major hubs are clearly visible a German one centered around HU Berlin / Heidelberg / Göttingen, an US-UK one centered around Harvard and Oxford, and a Russian one centered around Moscow SU.

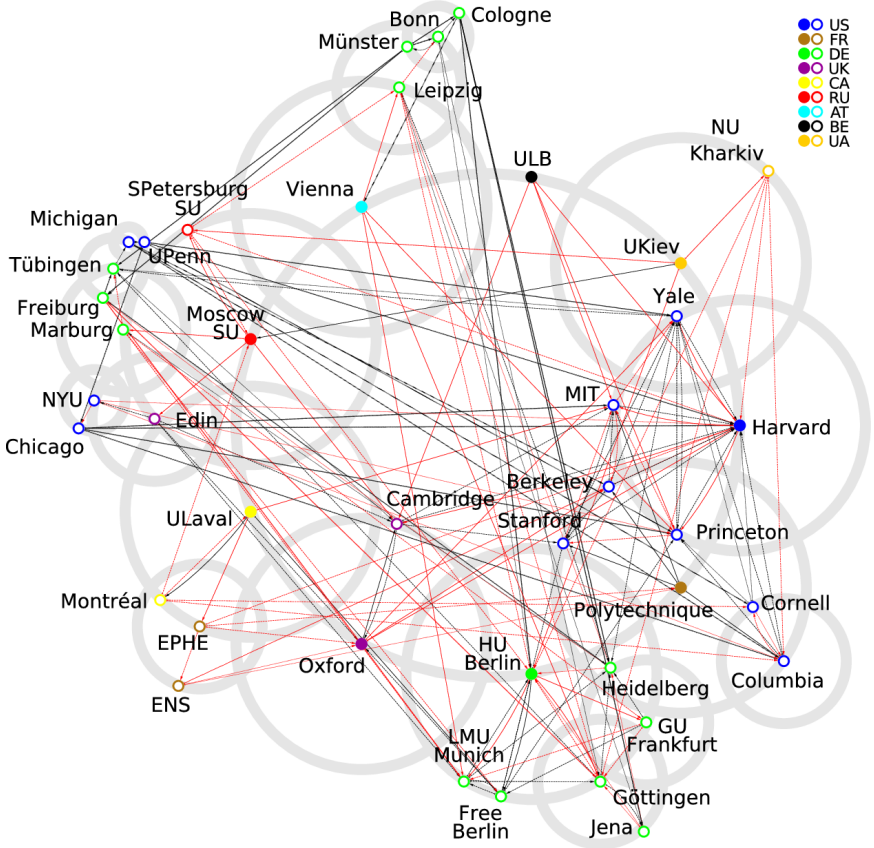

Fig. 18. Same as in Fig. 16 but from DEWIKI.

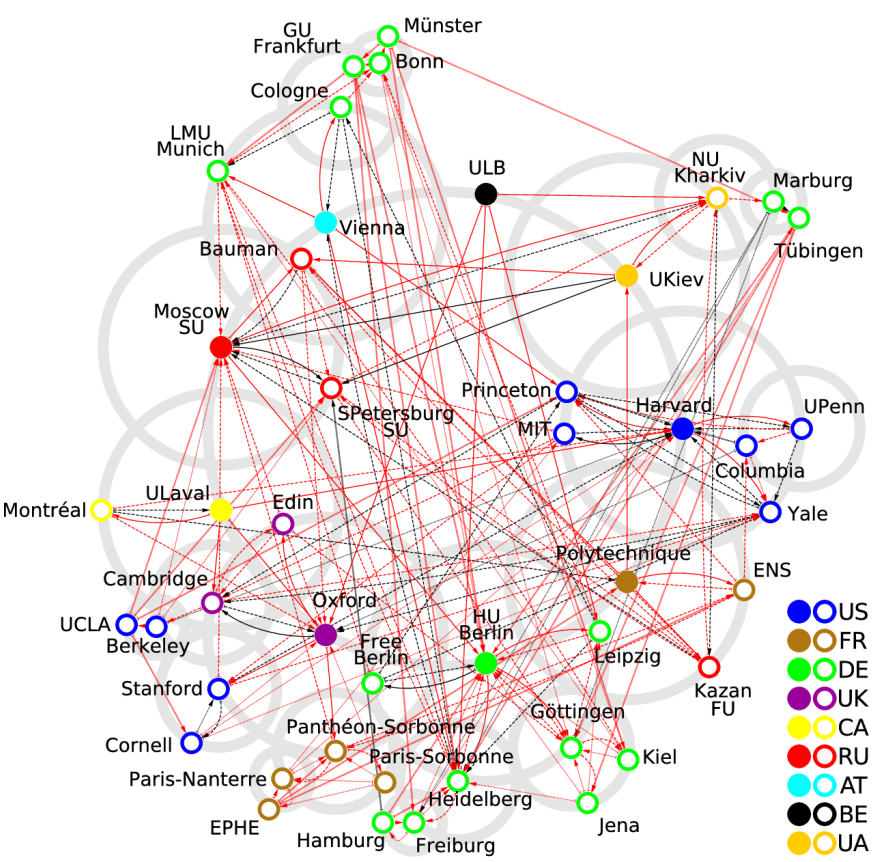

Fig. 19. Same as in Fig. 16 but from RUWIKI.

The analysis of this subsection allows to establish most close links between top world universities. It also shows the dominance of US and German universities.

\section{Reduced Google matrix averaged over 24 Wikipedia editions}

Above we have considered ranking and interactions from a view point of a given edition. Using the reduced Google 
matrix approach it is possible to perform an averaging over all 24 editions thus determining the averaged cultural view on selected universities. With this aim, for each of the 24 Wikipedia editions listed in Tab. 1, we select the subset of articles devoted to the 100 first PageRank universities of WRWU (see Tab. SI1). Then we compute the corresponding reduced Google matrix $\bar{G}_{\mathrm{R}}$ averaged over 24 Wikipedia editions. The averaging is defined by the relation

$$
\bar{G}_{\mathrm{R}}=\frac{1}{24} \sum_{E} G_{\mathrm{R}}^{(E)}
$$

where $G_{\mathrm{R}}^{(E)}$ is the reduced Google matrix (4) of the Wikipedia edition $E$. Each one of the 24 reduced Google matrices is written in the same basis corresponding to the ordered PageRank list of 100 universities. For a given edition $E$, reduced Google matrix entries corresponding to a link pointing toward an absent university in edition $E$ are set to 0 and reduced Google matrix columns corresponding to absent universities in edition $E$ are filled with 1/100 entries. These contributions from absent universities are added to the $G_{\mathrm{pr}}^{(E)}$ matrix components of the full reduced Google matrices $G_{\mathrm{R}}^{(E)}$.

We note that the averaging of $24 G_{\mathrm{R}}^{(E)}$ matrices with equal weights gives us again the reduced Google matrix which performs an averaging over different cultural views of 24 editions.

The PageRank vector computed from the averaged reduced Google matrix $\bar{G}_{\mathrm{R}}$ is presented in Tab. 10 . We see that the rank order is changed comparing to the $\Theta$ averaging (5) with the top 10 PageRank universities given in Tab. 2 (list of top 100 is given in Tab. SI1). We see that Harvard takes the first position instead of the third one in Tab. 2 and then Oxford and Cambridge are moved to second and third positions in Tab. 10. The top ten universities of Tab. 10 have overlap of $100 \%$ with PageRank WRWU of Tab. 2 and $90 \%$ with ARWU of Tab. 3. It can be discussed what ranking averaging over 24 cultural view of editions is more appropriate: with $\Theta$-averaging or with averaging of $G_{\mathrm{R}}^{(E)}$. We think that both approaches are useful: in $\Theta$-averaging all PageRanking vectors are completely independent while averaging of $G_{\mathrm{R}}^{(E)}$ introduces some additional links which are not present in certain network editions.

The obtained averaged Reduced Google matrix $\bar{G}_{\mathrm{R}}$ and its three components are shown in Fig. 20. In global the matrix structure is similar to those of individual editions discussed above. The component $\bar{G}_{\mathrm{pr}}$ has the dominant weight but it is rather close to the columns of $\mathrm{Pa}$ geRank vector and hence the interesting links are determined by the components $\bar{G}_{\mathrm{rr}}$ and $\bar{G}_{\mathrm{qr}}$ which have comparable weights. It is well seen that there are indirect links which are not present between direct ones.

From the matrix of direct and indirect links $\bar{G}_{\mathrm{rr}}+$ $\bar{G}_{\text {qrnd }}$ we construct the interaction friendship network between above considered 100 universities divided by certain groups. Such a network takes into account cultural views of all 24 editions. We now show all links by the same black color since after averaging over 24 editions there is a significant mixture of direct and indirect links.

In our first division, we mark universities by foundation time (century) periods: red for foundation years from 1000 to $1300 \mathrm{AD}$, blue from 1300 to $1600 \mathrm{AD}$, green from 1600 to 1800 AD and black from 1800 to 2000 AD. Each time period has its leader taken as a university with highest rank position in this period. The resulting network of friends in shown in Fig. 21. This network shows an interesting evolution of interactions between universities through 10 centuries: the cluster of universities founded in 11 th-13h centuries, marked in red, is formed mainly by UK and Italian universities (one from Spain, group leader is Oxford). This cluster transfers its influence via interaction and links to next 14th-16th centuries universities, marked in blue, which are mainly from northern countries including Scotland, Denmark, Germany, Sweden and Netherlands (group leader is Copenhagen). The influence of these universities is transfered to 17th-18th centuries universities, marked in green, being mainly near the blue cluster and located in the same countries with addition of Moscow in Russia, Tartu in Estonia, Helsinki in Finland;

Table 10. Top100 2017 WRWU ordered according to averaged reduced Google matrix.

\begin{tabular}{|c|c|c|c|c|c|}
\hline Rank & $\begin{array}{l}\text { PageRank } \\
\text { value }\end{array}$ & University & Rank & $\begin{array}{c}\text { PageRank } \\
\text { value }\end{array}$ & University \\
\hline 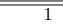 & 0.0633191 & " Harvard University & $\overline{51}$ & 0.00659655 & 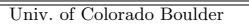 \\
\hline 2 & 0.0528587 & Univ. of Oxford & 52 & 0.00657266 & Univ. of Glasgow \\
\hline 3 & 0.0518905 & Univ. of Cambridge & 53 & 0.00636839 & Univ. of Toronto \\
\hline 4 & 0.0339304 & $\mathrm{MIT}^{a}$ & 54 & 0.0063255 & Stockholm University \\
\hline 5 & 0.0301911 & Columbia University & 55 & 0.00624184 & Univ. of Tübingen \\
\hline 6 & 0.0283041 & Yale University & 56 & 0.00609986 & Univ. of Texas at Austin \\
\hline 7 & 0.0261455 & Stanford University & 57 & 0.00593539 & Univ. of Virginia \\
\hline 8 & 0.024318 & UC Berkeley $^{b}$ & 58 & 0.00584412 & Imperial College London \\
\hline 9 & 0.0229394 & Princeton University & 59 & 0.00582829 & Carnegie Mellon University \\
\hline 10 & 0.0215136 & Univ. of Chicago & 60 & 0.00579437 & Univ. of Bonn \\
\hline 11 & 0.0197203 & Univ. of Copenhagen & 61 & 0.00570673 & Univ. of Minnesota \\
\hline 12 & 0.0168679 & HU Berlin ${ }^{c}$ & 62 & 0.00567465 & Keio University \\
\hline 13 & 0.0160439 & Uppsala university & 63 & 0.00557384 & Univ. of Helsinki \\
\hline 14 & 0.0148231 & Univ. of Tokyo & 64 & 0.00548871 & King's College London \\
\hline 15 & 0.0135633 & Moscow State University & 65 & 0.0054485 & Univ. of Florida \\
\hline 16 & 0.0127305 & Cornell University & 66 & 0.00538279 & Univ. of Zurich \\
\hline 17 & 0.0126064 & $\mathrm{HUJI}^{d}$ & 67 & 0.00536546 & Univ. of Manchester \\
\hline 18 & 0.0125732 & Univ. of Pennsylvania & 68 & 0.00523928 & McGill University \\
\hline 19 & 0.0120329 & $\mathrm{UCLA}^{e}$ & 69 & 0.00507791 & Free University of Berlin \\
\hline 20 & 0.011732 & Leiden University & 70 & 0.00505635 & Univ. of Washington \\
\hline 21 & 0.011246 & Caltech $^{f}$ & 71 & 0.00505447 & Univ. of Illinois U.-C. \\
\hline 22 & 0.0112404 & New York University & 72 & 0.00497258 & Brown University \\
\hline 23 & 0.0112273 & Univ. of Vienna & 73 & 0.00491403 & Univ. of Wisconsin-Madison \\
\hline 24 & 0.0104997 & Univ. of Edinburgh & 74 & 0.00485964 & Northwestern University \\
\hline 25 & 0.0103698 & Jagiellonian University & 75 & 0.00480294 & Univ. of Coimbra \\
\hline 26 & 0.0101557 & Univ. of Bologna & 76 & 0.00479832 & Univ. of Oslo \\
\hline 27 & 0.0100089 & Univ. of Göttingen & 77 & 0.00477973 & Univ. of Padua \\
\hline 28 & 0.00987766 & Heidelberg University & 78 & 0.00476805 & Georgetown University \\
\hline 29 & 0.00982921 & Univ. of Michigan & 79 & 0.00475634 & $\mathrm{UNAM}^{l}$ \\
\hline 30 & 0.00974263 & Lund University & 80 & 0.00468635 & Boston University \\
\hline 31 & 0.00929623 & $\mathrm{LSE}^{g}$ & 81 & 0.0045985 & Ohio State University \\
\hline 32 & 0.00918967 & Johns Hopkins University & 82 & 0.00458516 & Michigan State University \\
\hline 33 & 0.00909002 & Univ. of Warsaw & 83 & 0.00452351 & $\begin{array}{l}\text { Univ. of Geneva } \\
\text { S }\end{array}$ \\
\hline 34 & 0.00902656 & Seoul National University & 84 & 0.00451385 & Univ. of Marburg \\
\hline 35 & 0.00877768 & Leipzig University & 85 & 0.00433353 & Univ. of Salamanca \\
\hline 36 & 0.00832413 & Univ. of Munich ${ }^{h}$ & 86 & 0.0042273 & Univ. of Freiburg \\
\hline 37 & 0.00791791 & Waseda University & 87 & 0.00418341 & Univ. of Arizona \\
\hline 38 & 0.0076835 & Univ. College London & 88 & 0.00417181 & Univ. of Jena \\
\hline 39 & 0.00751886 & Duke University & 89 & 0.00415139 & $\mathrm{MLU}^{m}$ \\
\hline 40 & 0.00718132 & Sapienza $^{i}$ & 90 & 0.00401368 & Univ. of St Andrews \\
\hline 41 & 0.00711981 & ETH Zurich & 91 & 0.00398415 & TU Berlin ${ }^{n}$ \\
\hline 42 & 0.0071081 & $\mathrm{USC}^{j}$ & 92 & 0.00391916 & UNC Chapel Hill $^{\circ}$ \\
\hline 43 & 0.00693105 & École Polytechnique & 93 & 0.00390789 & Univ. of Tartu \\
\hline 44 & 0.00692597 & Peking University & 94 & 0.00388656 & TU Munich $^{p}$ \\
\hline 45 & 0.00682986 & Al-Azhar University & 95 & 0.00385376 & Univ. of Sydney \\
\hline 46 & 0.00682254 & École Normale Supérieure & 96 & 0.00384341 & UC San Diego $^{q}$ \\
\hline 47 & 0.00680075 & Kyoto University & 97 & 0.00371085 & Trinity College, Dublin \\
\hline 48 & 0.00666809 & Charles University & 98 & 0.00368454 & Indiana University \\
\hline 49 & 0.00666454 & $\mathrm{SPbU}^{k}$ & 99 & 0.00355122 & University of Notre Dame \\
\hline 50 & 0.00662585 & Utrecht University & 100 & 0.00353878 & University of Kiel \\
\hline \multicolumn{6}{|c|}{$\begin{array}{l}{ }^{a} \text { Massachusetts Institute of Technology, }{ }^{b} \text { University of California, Berkeley, }{ }^{c} \text { Humboldt University of } \\
\text { Berlin, }{ }^{d} \text { Hebrew University of Jerusalem, }{ }^{e} \text { University of California, Los Angeles, }{ }^{f} \text { California Institute } \\
\text { of Technology, }{ }^{g} \text { London School of Economics, }{ }^{h} \text { Ludwig Maximilian University of Munich, }{ }^{i} \text { Sapienza } \\
\text { University of Rome, }{ }^{j} \text { University of Southern California, }{ }^{k} \text { Saint Petersburg State University, }{ }^{l} \text { National } \\
\text { Autonomous University of Mexico, }{ }^{m} \text { Martin Luther University of Halle-Wittenberg, }{ }^{n} \text { Technical Univer- } \\
\text { sity of Berlin, }{ }^{o} \text { University of North Carolina at Chapel Hill, }{ }^{p} \text { Technical University of Munich, }{ }^{q} \text { University } \\
\text { of California, San Diego }\end{array}$} \\
\hline
\end{tabular}




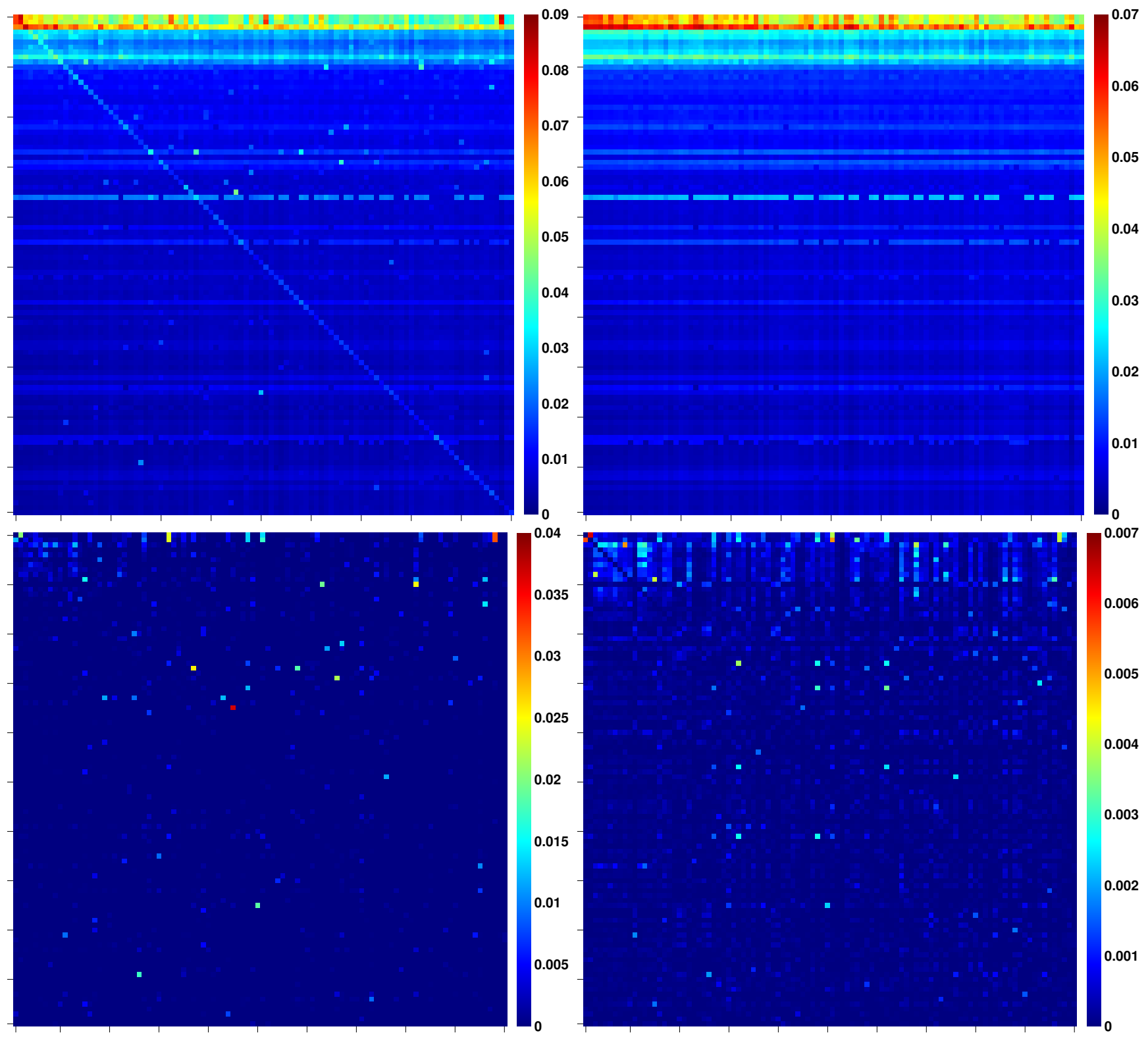

Fig. 20. Reduced Google matrix $\bar{G}_{\mathrm{R}}$ for PageRank top 100 universities in WRWU (top 10 is in Tab. 2, top 100 is in Tab. SI1) averaged over 24 Wikipedia editions. Matrix entries correspond to universities ordered according the top 100 WRWU PageRank order. The full reduced Google matrix $\bar{G}_{\mathrm{R}}$ is presented in top left panel, $\bar{G}_{\mathrm{pr}}$ in top right panel, $\bar{G}_{\mathrm{rr}}$ in bottom left panel, and $\bar{G}_{\mathrm{qrnd}}$ in bottom right panel. The matrix weights are $W_{\mathrm{R}}=1, W_{\mathrm{pr}}=0.957, W_{\mathrm{rr}}=0.019, W_{\mathrm{qr}}=0.024, W_{\mathrm{qrnd}}=0.015$.

another group of green universities of this time period is linked with Oxford and Cambridge and is located mainly on US east coast (Harvard, Yale, Princeton; Columbia is directly linked to Cambridge). The university of next centuries 19th-20th, marked in black (MIT is group leader), are mainly located in US but new universities of this time period appear also in Japan (Tokyo, Kyoto), Egypt (AlAzhar), Germany (TU Munich, TU Berlin, Free Berlin) and Sweeden (Stockholm). Thus the obtained friendship network provides a compact description of world universities development through 10 centuries taking into account the balanced view of 24 cultures presented by Wikipedia editions.
In our second division we mark universities by continent location: blue for America, red for Europe, green for Asia (Russia is attributed to Asia), yellow for Oceania and black for Africa. Again color group leaders are marked by full circles. The friendship network obtained from $\bar{G}_{\text {rr }}+\bar{G}_{\text {qrnd }}$ is shown in Fig. 22 . The network has two large clusters of European universities (red) and US universities (blue). The group of university in Asia (green) is mainly linked between themselves having secondary links with Europe and US. Oceania (Sydney) and Africa (AlAzhar) are represented only by one university. This network structure clearly shows the influence of European 
$0[1000,1300[$

$0[1300,1600[$

$0[1600,1800[$

๑०[1800,2000]

Moscow Helsinki

Toronto of Tartu

Stockholm of Un Utrecht

Uppsala A $^{\circ}=0$ jHopkins

cöttygeno Heidelorg

MLuther

Hálle-Witteingerg

agen Padua

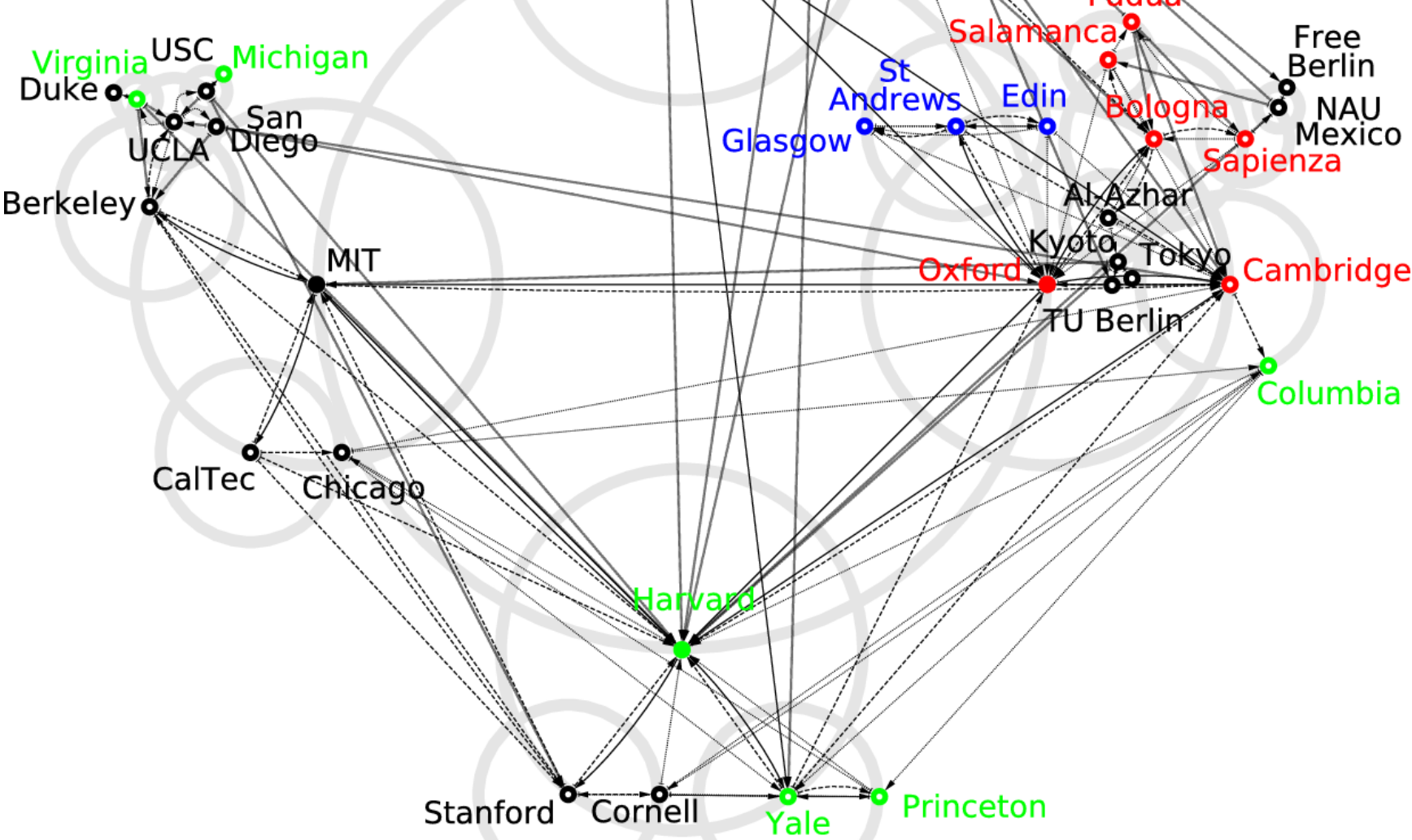

Fig. 21. Century reduced friendship network constructed for universities of PageRank top 100 list of WRWU (see Tab. SI1, top 10 are in Tab. 2), computed from $\bar{G}_{\mathrm{rr}}+\bar{G}_{\mathrm{qrnd}}$ averaged over 24 Wikipedia editions. Color marks university founded at the same time (century) period given in years; color filled circles are time period leaders, open circles of the same color are universities from the same time period. We show 5 friendship levels (gray circles). Links originating from 1st level universities are presented by solid lines, from 2nd level by dashed lines, from 3rd level by doted lines, and from 4th or 5th level by "V" symbol lines.

and US universities with emerging group of new group of Asian universities with strong internal links.

\section{Discussion}

In this work we performed analysis of ranking and interactions of world universities from directed networks of 24 Wikipedia editions dated by May 2017. Our results show that obtained WRWU2017 with PageRank algorithm averaged over 24 editions gives a reliable ranking of universi- ties with $60 \%$ overlap with top 100 of ARWU2017 (Shanghai ranking) [3]. At the same time WRWU2017 highlights in a stronger way the significance of historical path of a given university over centuries. There are certain changes in WRWU2017 version comparing to WRWU2013 version demonstrating appearance of new universities with time evolving and with the increase of the number of Wikipedia articles in the 24 selected editions. A comparison of WRWU and ARWU ranking positions for specific uni- 


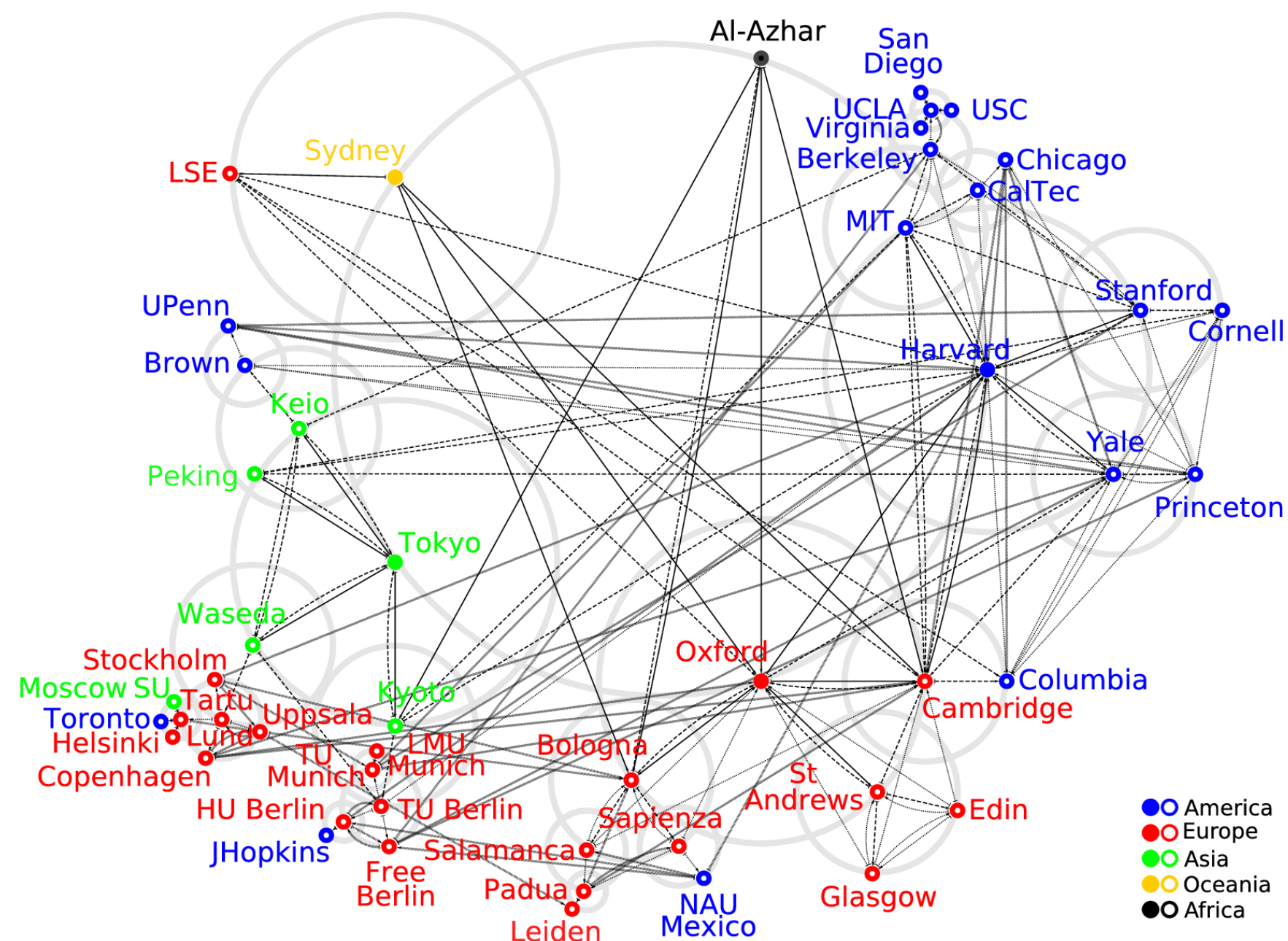

Fig. 22. Continent reduced friendship network constructed for universities of PageRank top 100 list of WRWU (see Tab. SI1, top 10 are in Tab. 2), computed from $\bar{G}_{\text {rr }}+\bar{G}_{\text {qrnd }}$ averaged over 24 Wikipedia editions. Color marks university of the same continent; color filled circles are continent leaders, open circles of the same color are universities from the same continent. We obtain 5 friendship levels (gray circles). Links originating from 1st level universities are presented by solid lines, from $2 \mathrm{nd}$ level by dashed lines, from 3rd level by doted lines, and from 4th or 5th level by "\" symbol lines.

versities (e.g. Rice University) shows that the Wikipedia visibility can be significantly improved in certain cases.

We also performed an additional analysis based on the reduced Google matrix (REGOMAX) algorithm [26,27]. This approach allowed us to establish direct and indirect links between universities and world countries. As a result we obtain the sensitivity and influence of specific universities on world countries as it is seen by Wikipedia. The REGOMAX method allows to perform a democratic and uniform averaging over cultural views of 24 language editions and obtain a balanced cultural view on the interactions of top world universities through ten centuries of their historical development as well as their influence over continents.

Finally we stress that the WRWU method is independent of various personal opinions being based on purely mathematical and statistical analysis of the Wikipedia database. We think that this approach can be very complimentary to ARWU and other university rankings. We note that Wikipedia articles of universities usually appear in the top line (or lines) of Google search. As a result the world visibility of a given university can be publicly and freely broadcast all over the world increasing visibility of certain universities. We estimate that the improvement of Wikipedia articles of certain universities (e.g. we found a low visibility of French universities) can be an efficient way to increase their world visibility, attractivity and influence. Such an improvement is rather inexpensive and can be performed by a small group of researchers and students having knowledge in languages, history, computer and network sciences. We think that this approach can be complementary to various government projects which aim to increase visibility of national universities (like e.g. [4, $5])$.

\section{Acknowledgments}

This work was supported by the French "Investissements d'Avenir" program, project ISITE-BFC (contract ANR-15-IDEX0003) and by the Bourgogne Franche-Comté Region 2017-2020 
APEX project (conventions 2017Y-06426, 2017Y-06413, 2017Y07534; see http://perso.utinam.cnrs.fr/ lages/apex/). The research of DLS is supported in part by the Programme Investissements d'Avenir ANR-11-IDEX-0002-02, reference ANR10-LABX-0037-NEXT (project THETRACOM).

\section{References}

1. UNESCO Liaison Office 24 July 2017, New York Office, Available: ww. unesco.org/new/ en/unesco-liaison-office-in-new-york/ about-this-office/single-view/news/unesco_ emphasizes_the_role_of_universities_and_higher_ educat/. Accessed July 2018

2. E. Hazelkorn, Rankings and the Reshaping of Higher Education: The Battle for World-Class Excellence, Palgrave Macmillan, New York (2015)

3. Academic Ranking of World Universities. Available: http: //www. shanghairanking. com/. Accessed July 2018

4. Enseignement supérieur et recherche, Investissements d'avenir. Available: http://www . enseignementsup-recherche.gouv.fr/pid24578/ investissements-d-avenir.html. Accessed July 2018

5. Russian Academic Excellence Project. Available: http:// 5top100.ru/. Accessed July 2018

6. Times Higher Education World University Ranking. Available: https://www.timeshighereducation.com. Accessed July 2018

7. U-Multirank of European Union. Available: http://www . umultirank.org/. Accessed July 2018

8. IREG Observatory on Academic Ranking and Excellence. Available: http://ireg-observatory.org/en/. Accessed July 2018

9. H. Jöns and M. Hoyler, Global geographies of higher education: The perspective of world university rankings, Geoforum 46, 45 (2013)

10. A. Rauhvargers, Global University Rankings and Their Impact - Report II, European University Association (2013) ISBN: 9789078997412

11. D. Docampo and L. Cram, On the internal dynamics of the Shanghai ranking, Scientometrics 98, 1347 (2014)

12. A.O. Zhirov, O.V. Zhirov and D.L. Shepelyansky, Twodimensional ranking of Wikipedia articles, Eur. Phys. J. B 77, 523 (2010)

13. Y.-H. Eom, K.M. Frahm, A. Benczur and D.L. Shepelyansky, Time evolution of Wikipedia network ranking, Eur. Phys. J. B 86, 492 (2013)

14. J.Lages, A.Patt and D.L.Shepelyansky, Wikipedia ranking of world universities, Eur. Phys. J. B 89, 69 (2016)

15. Y.-H. Eom, P.Aragon, D.Laniado, A.Kaltenbrunner, S.Vigna and D.L. Shepelyansky, Interactions of cultures and top people of Wikipedia from ranking of 24 language editions, PLoS ONE 10(3), e0114825 (2015)

16. EC FET Open project NADINE Available: www. quantware.ups-tlse.fr/FETNADINE/. Accessed July 2018

17. S. Brin and L. Page, The anatomy of a large-scale hypertextual Web search engine, Computer Networks and ISDN Systems 30, 107 (1998).

18. A.M. Langville and C.D. Meyer, Google's PageRank and beyond: the science of search engine rankings, Princeton University Press, Princeton (2006)
19. L. Ermann, K.M. Frahm and D.L. Shepelyansky, Google matrix analysis of directed networks, Rev. Mod. Phys. 87, $1261(2015)$

20. L. Ermann, K.M. Frahm and D.L. Shepelyansky, Google matrix, Scholarpedia 11(11), 30944 (2016)

21. R.A. Pagel (2016)
http://librarylearningspace.com/ ruths-rankings-17-wikipedia-google-scholar-sources -university-rankings-influence-popularity -open-bibliometrics/. Accessed July 2018

22. Web page WRWU 2013, Available: http://perso. utinam.cnrs.fr/ lages/datasets/WRWU/. Accessed July 2018

23. G. Katz and L. Rokach, Wikiometrics: a Wikipedia based ranking system, World Wide Web 20(6), 1153 (2017)

24. K. Ban, M. Perc and Z. Levnajic, Robust clustering of languages across Wikipedia growth, R. Soc. open sci. 4, 171217 (2017)

25. K.M. Frahm and D.L. Shepelyansky, Wikipedia networks of 24 editions of 2017, Available: http://www .quantware . ups-tlse.fr/QWLIB/24wiki2017. Accessed July 2018

26. K.M. Frahm and D.L. Shepelyansky, Reduced Google matrix, arXiv:1602.02394[physics.soc] (2016)

27. K.M. Frahm, K. Jaffrès-Runser and D.L. Shepelyansky, Wikipedia mining of hidden links between political leaders, Eur. Phys. J. B 89, 269 (2016)

28. S. El Zant, K.M. Frahm, K. Jaffres-Runser and D.L. Shepelyansky, Analysis of world terror networks from the reduced Google matrix of Wikipedia, Eur. Phys. J. B 91, 7 (2018)

29. J. Lages, D.L. Shepelyansky and A. Zinovyev, nferring hidden causal relations between pathway members using reduced Google matrix of directed biological networks, PLoS ONE 13(1), e0190812 (2018)

30. Web page WRWU 2017, Available: http://perso. utinam.cnrs.fr/ lages/datasets/WRWU17/. Accessed July 2018

31. A.D. Chepelianskii, Towards physical laws for software architecture, arXiv:1003.5455 [cs.SE] (2010)

32. Country codes ISO 3166-1 alpha-2. Available: https:// en.wikipedia.org/wiki/ISO_3166-1_alpha-2. Accessed July 2018

33. Jenks natural breaks optimization entry in English Wikipedia, Available https://en.wikipedia.org/wiki/Jenks_ natural_breaks_optimization. Accessed June 2018

34. S. El Zant, K. Jaffres-Runser, K.M. Frahm and D.L. Shepelyansky, Interactions and influence of world painters from the reduced Google matrix of Wikipedia networks, PLoS ONE 13(8), e0201397 (2018)

35. P. Shannon, A. Markiel, O. Ozier, N.S. Baliga, J.T. Wang, D. Ramage, N. Amin, B. Schwikowski and T. Ideker, Cytoscape: a software environment for integrated models of biomolecular interaction networks, Genome Research 13(11), 2498-504 (2003) 


\section{Supplementary Information: World influence and interactions of universities from Wikipedia networks}

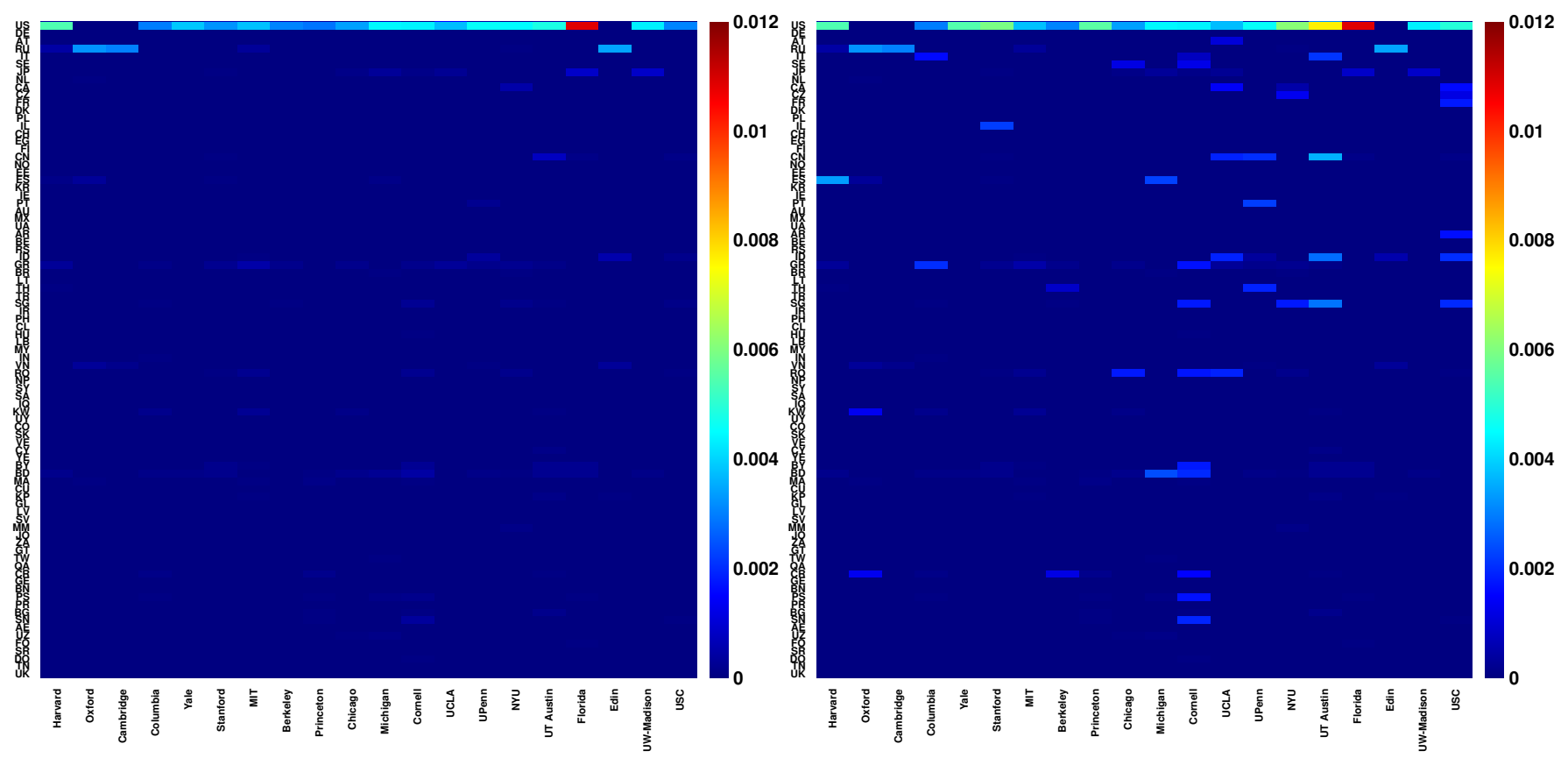

Fig. SI1. University to Country sector of the $G_{\mathrm{qr}}$ matrix (left panel) and of the $G_{\mathrm{rr}}+G_{\mathrm{qrnd}}$ composite matrix (right panel). See Fig. 5, bottom left (right) panel for the complete $G_{\mathrm{rr}}\left(G_{\mathrm{qr}}\right)$ matrix.

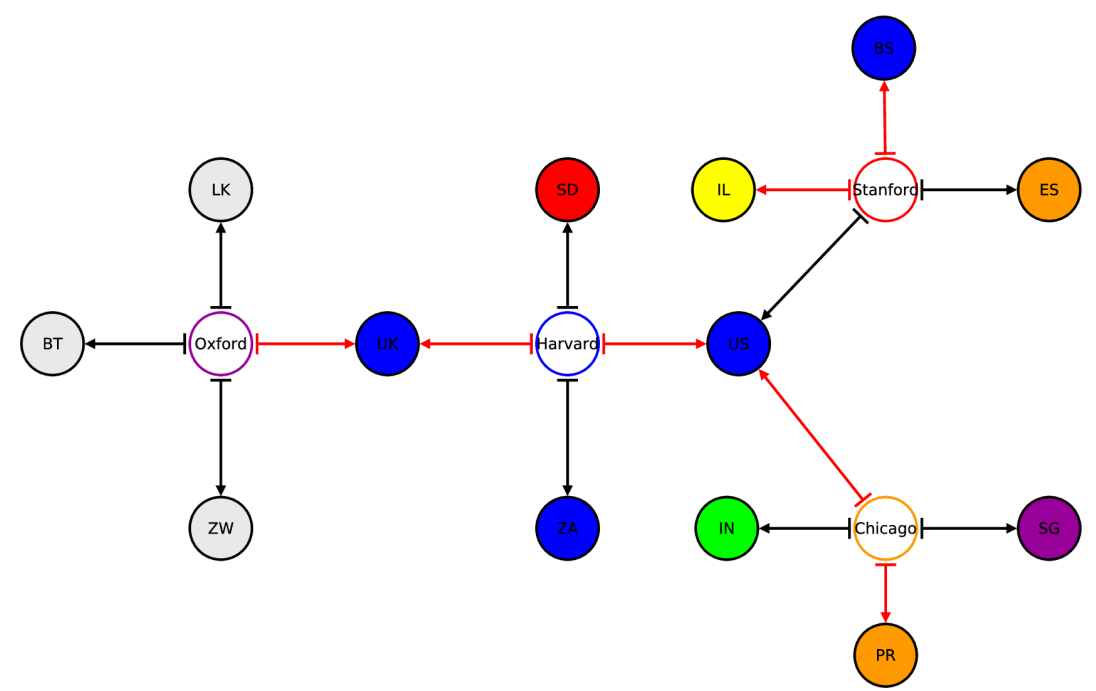

Fig. SI2. Reduced network from $G_{\mathrm{rr}}+G_{\mathrm{qrnd}}$ associated to the top20 ENWRWU and 240 countries listed in Tab. SI4. For each regional leaders, Stanford University, University of Chicago, Harvard University, University of Oxford, the four strongest links to one of the 240 countries are presented. Universities (countries) are represented by empty (full) nodes. The color code for countries depends on the main spoken language: blue for English, red for Arabic, orange for Spanish, violet for Chinese, green for Hindi, yellow for Hebrew, and gray for others. Red links are purely hidden links and black ones are at least present in the adjacency matrix. 


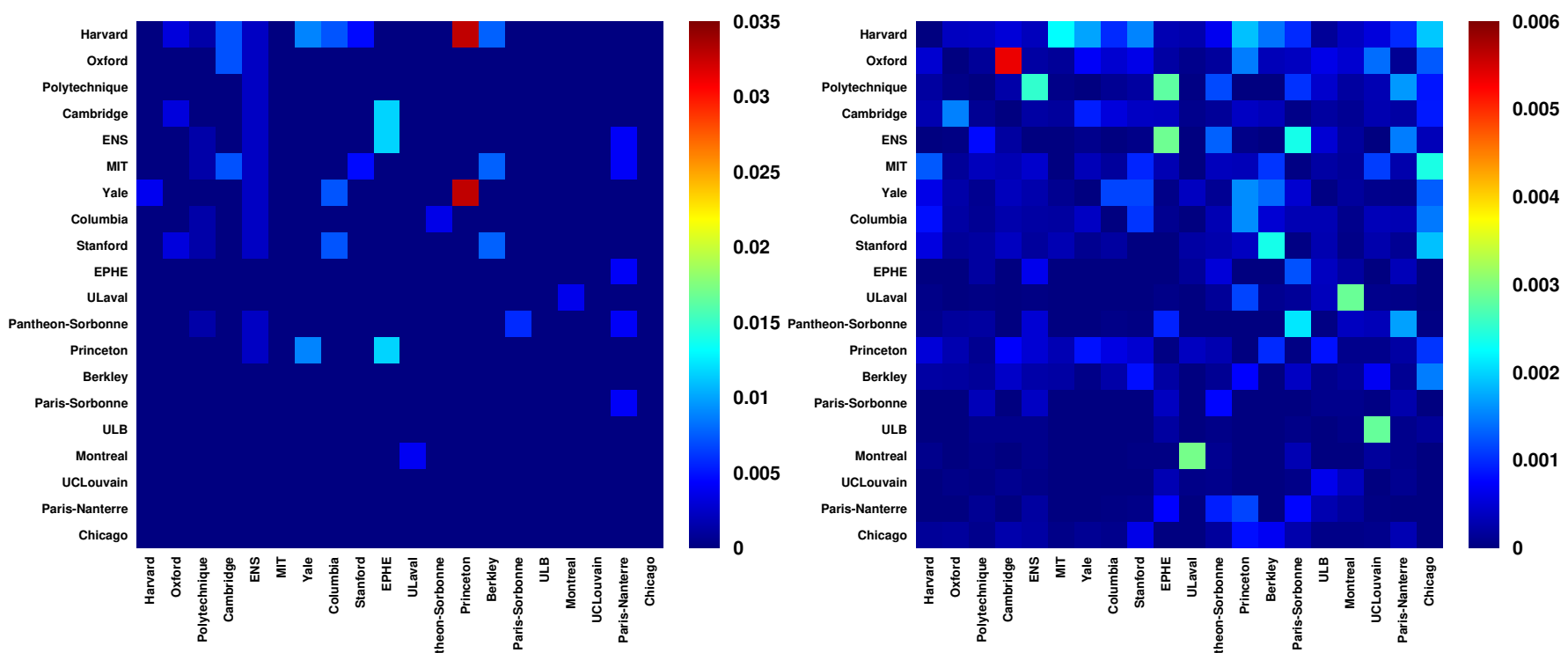

Fig. SI3. Matrices $G_{\mathrm{rr}}$ (left panel) and $G_{\text {qrnd }}$ (right panel) for top20 FRWRWU (Tab. 6). The matrix weights are $W_{\text {rr }}=0.01404$ and $W_{\text {qrnd }}=0.00746$.

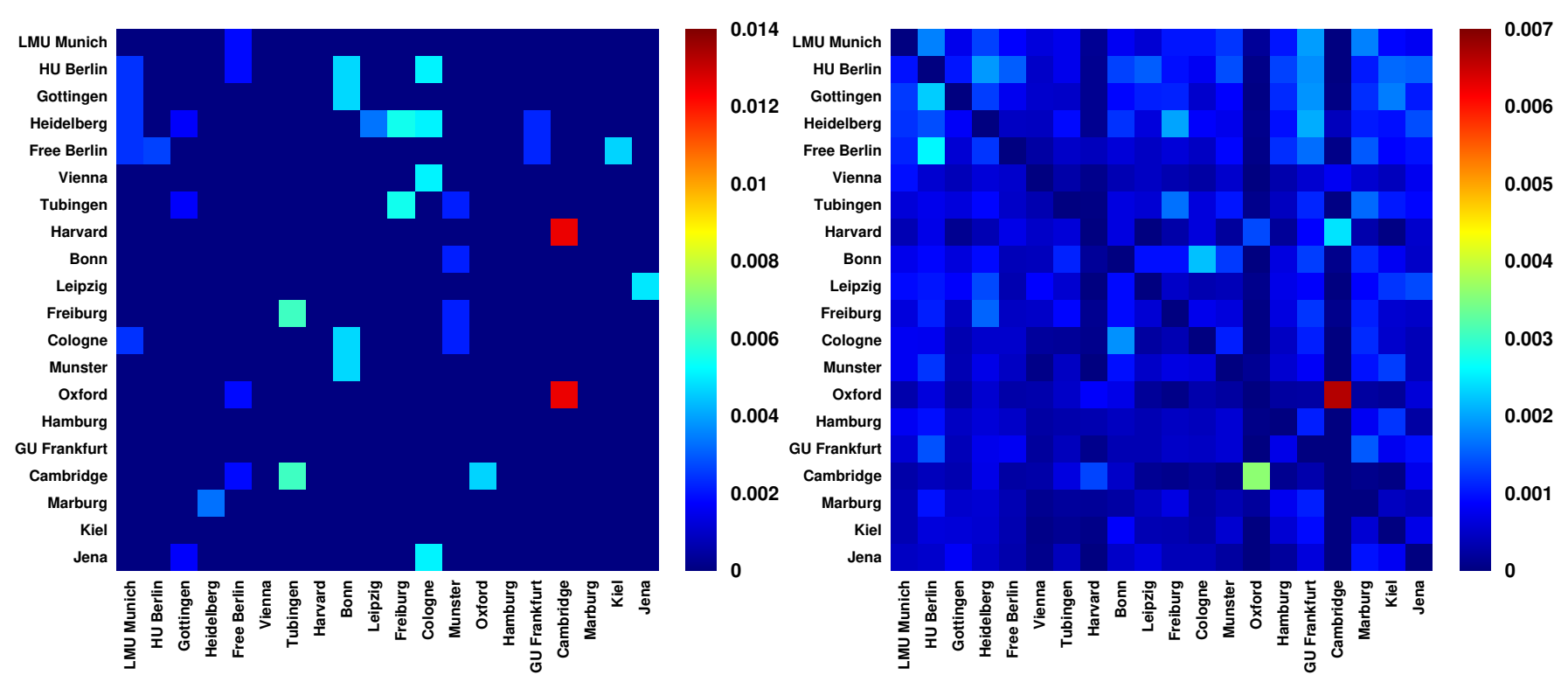

Fig. SI4. Matrices $G_{\mathrm{rr}}$ (left panel) and $G_{\mathrm{qrnd}}$ (right panel) for top20 DEWRWU (Tab. 7). The matrix weights are $W_{\mathrm{rr}}=0.00746$ and $W_{\text {qrnd }}=0.01280$. 


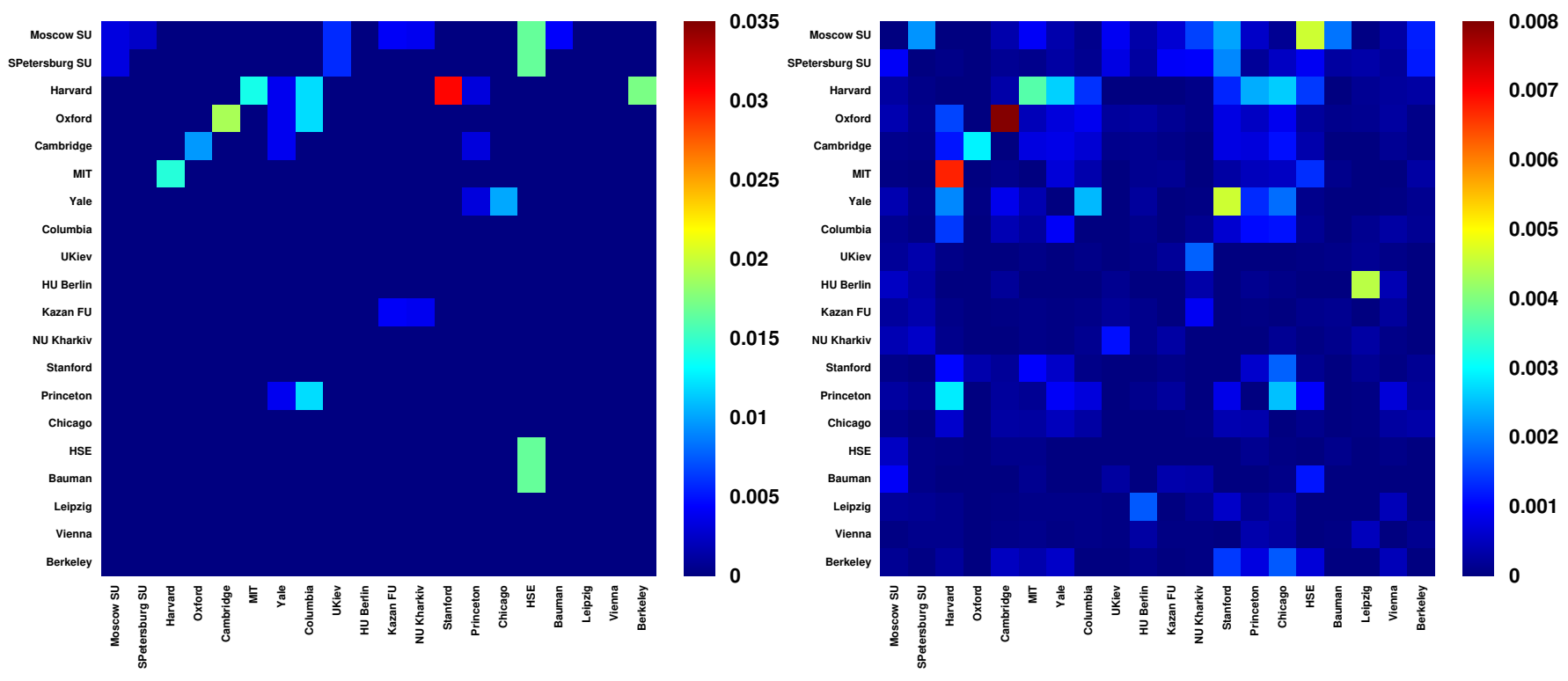

Fig. SI5. Matrices $G_{\mathrm{rr}}$ (left panel) and $G_{\mathrm{qrnd}}$ (right panel) for top20 RUWRWU (Tab. 8). The matrix weights are $W_{\mathrm{rr}}=0.01417$ and $W_{\text {qrnd }}=0.00798$. 


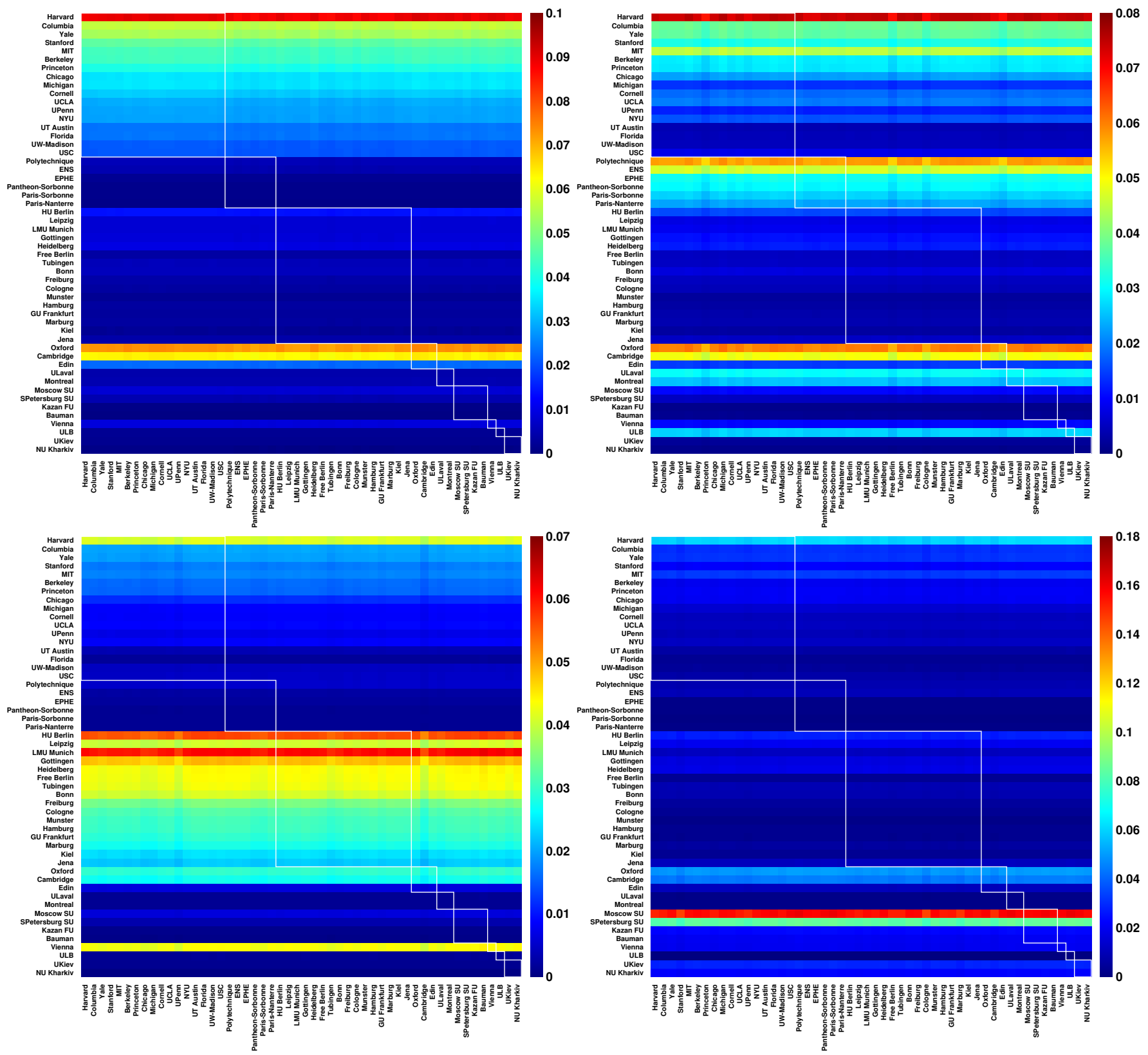

Fig. SI6. $G_{\text {pr }}$ part of the reduced Google matrix $G_{\mathrm{R}}$ for universities listed in Tab. 9 computed from EN (top left), FR (top right), $\mathrm{DE}$ (bottom left), and RU (bottom right) Wikipedia editions. Matrix weights are $W_{\mathrm{pr}} \simeq 0.956(\mathrm{EN}), 0.966$ (FR), 0.96 (DE) and $0.963(\mathrm{RU})$. 


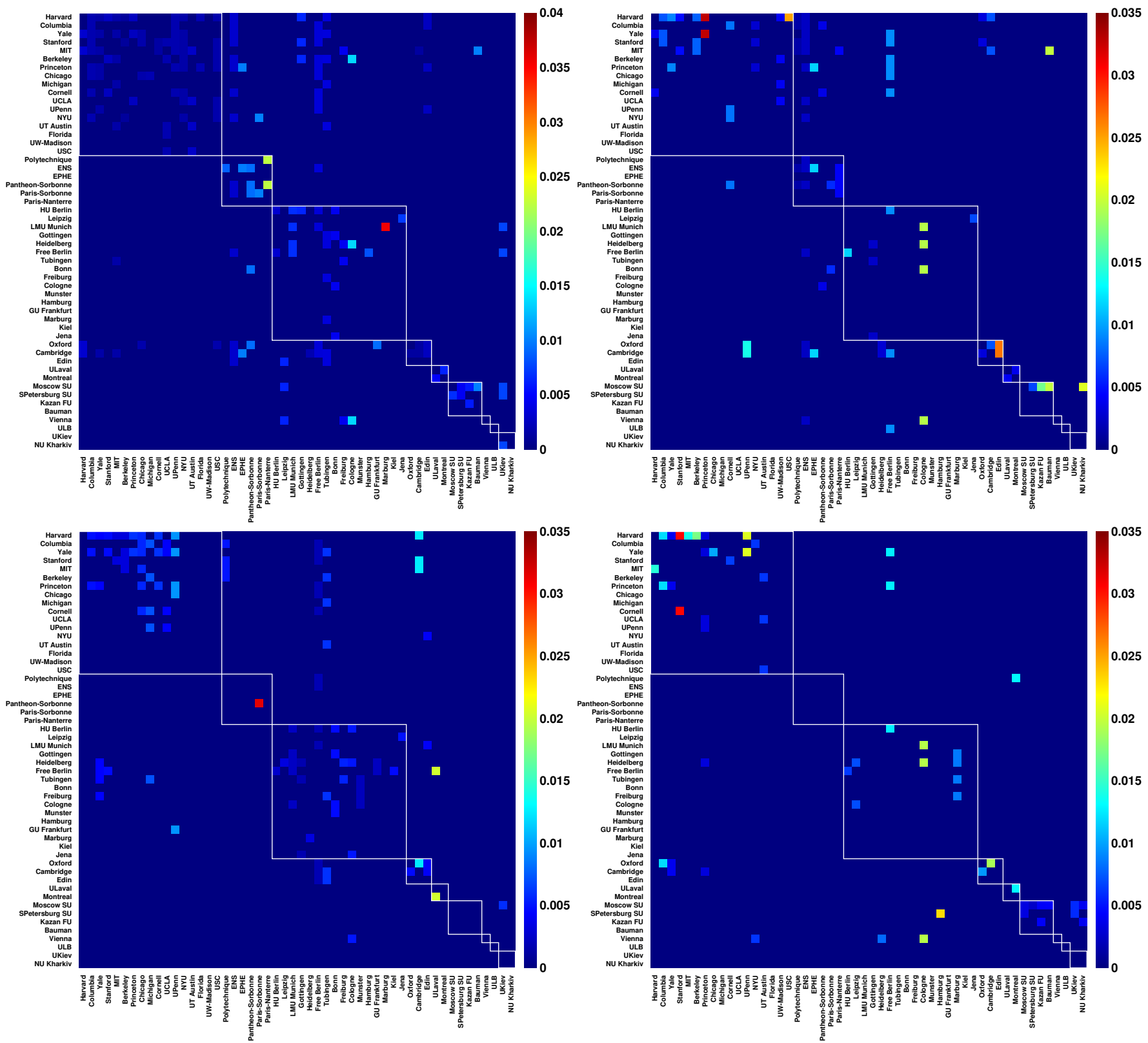

Fig. SI7. $G_{\mathrm{rr}}$ part of the reduced Google matrix $G_{\mathrm{R}}$ for universities listed in Tab. 9 computed from EN (top left), FR (top right), DE (bottom left), and RU (bottom right) Wikipedia editions. Matrix weights are $W_{\mathrm{rr}} \simeq 0.0148$ (EN), $0.0144(\mathrm{FR})$, 0.0111 (DE), 0.0105 (RU). 

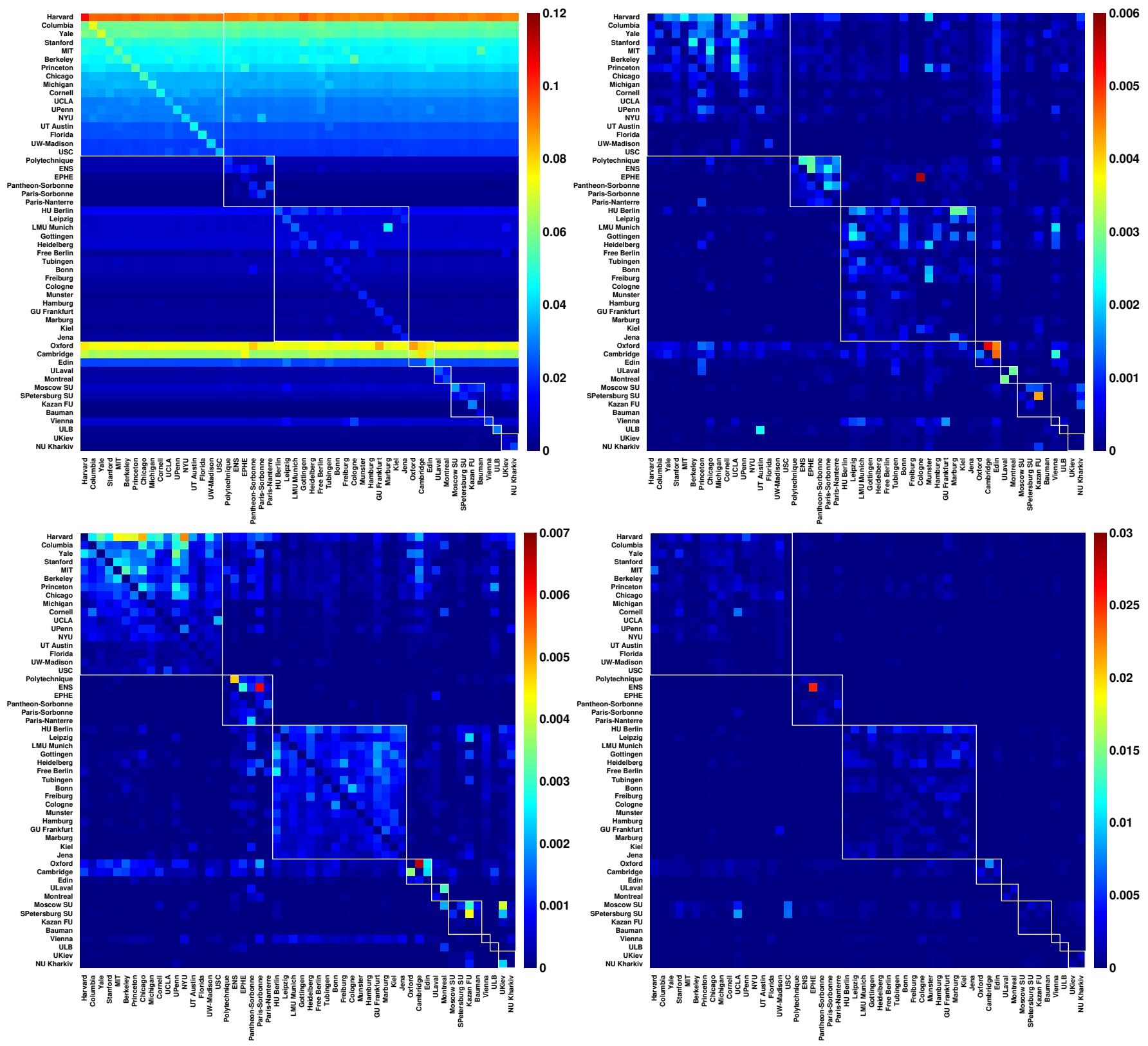

Fig. SI8. $G_{\mathrm{qr}}$ part of the reduced Google matrix $G_{\mathrm{R}}$ for universities listed in Tab. 9 computed from EN (top left), FR (top right), DE (bottom left), and RU (bottom right) Wikipedia editions. Matrix weights are $W_{\mathrm{qr}} \simeq 0.0153$ (EN), 0.00923 (FR), 0.015 (DE), $0.0138(\mathrm{RU})$. 
Table SI1. List of the first 100 universities of the 2017 Wikipedia Ranking of World Universities using PageRank algorithm. For a given university, the score $\Theta_{P R}$ is defined by (5), $N_{a}$ is the number of appearances in the top 100 lists of Wikipedia editions, CC is the country code, LC is the language code, and FC is the foundation century.

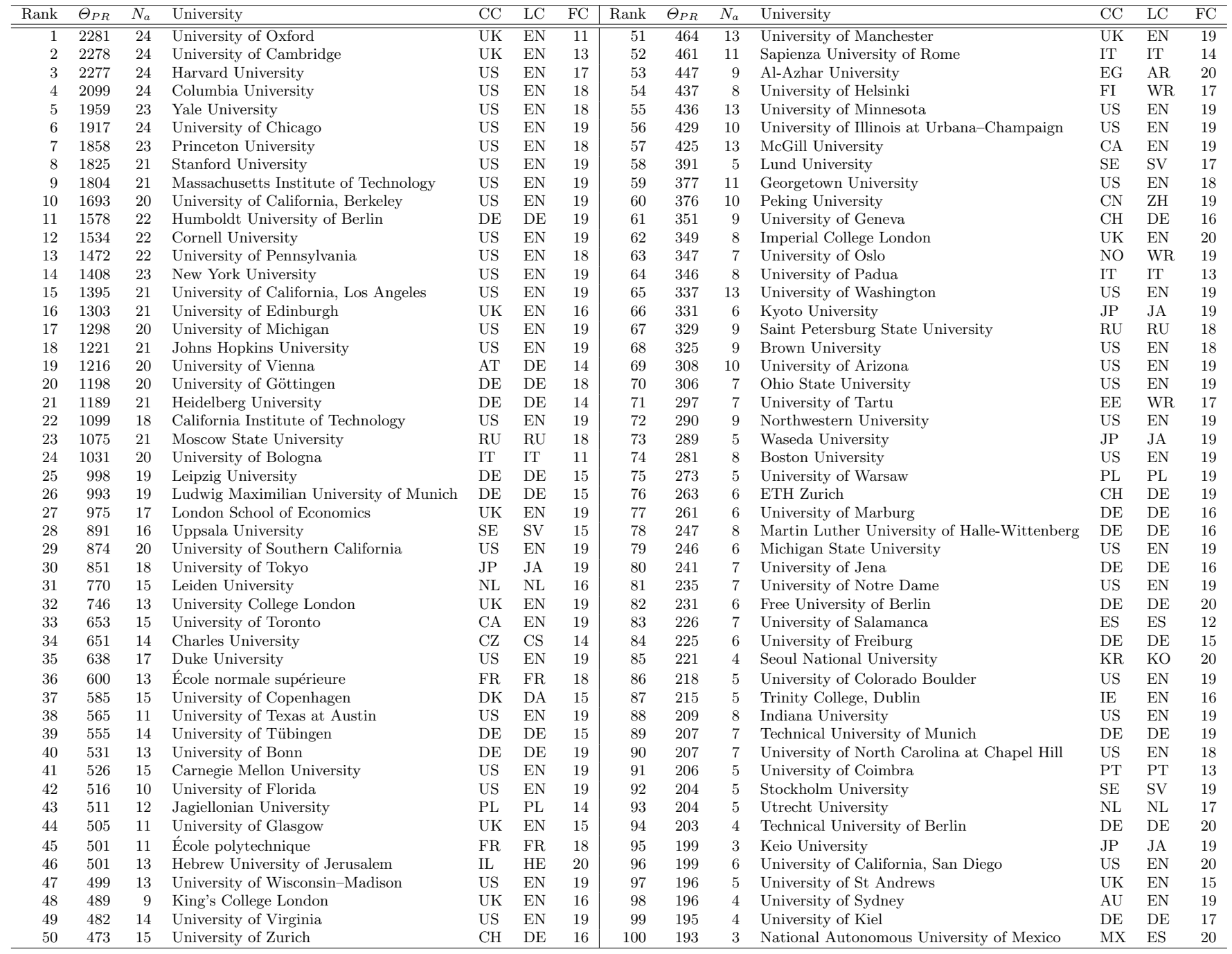


Table SI2. List of the first 100 universities of the 2017 Academic Ranking of World Universities (ARWU). CC is the country code, LC is the language code, and FC is the foundation century.

\begin{tabular}{|c|c|c|c|c|c|c|c|c|c|}
\hline Rank & University & $\mathrm{CC}$ & $\mathrm{LC}$ & $\mathrm{FC}$ & Rank & University & $\mathrm{CC}$ & $\mathrm{LC}$ & FC \\
\hline 1 & Harvard University & US & $\overline{\mathrm{EN}}$ & 17 & 51 & University of Texas at Austin & US & EN & 19 \\
\hline 2 & Stanford University & US & EN & 19 & 52 & Vanderbilt University & US & EN & 19 \\
\hline 3 & University of Cambridge & UK & EN & 13 & 53 & University of Maryland, College Park & US & $\mathrm{EN}$ & 19 \\
\hline 4 & Massachusetts Institute of Technology & US & EN & 19 & 54 & University of Southern California & US & $\mathrm{EN}$ & 19 \\
\hline 5 & University of California, Berkeley & US & EN & 19 & 55 & University of Queensland & $\mathrm{AU}$ & EN & 20 \\
\hline 6 & Princeton University & US & EN & 18 & 56 & University of Helsinki & FI & WR & 17 \\
\hline 7 & University of Oxford & UK & EN & 18 & 57 & Ludwig Maximilian University of Munich & $\mathrm{DE}$ & $\mathrm{DE}$ & 15 \\
\hline 8 & Columbia University & US & EN & 18 & 58 & University of Zurich & $\mathrm{CH}$ & $\mathrm{DE}$ & 16 \\
\hline 9 & California Institute of Technology & US & EN & 19 & 59 & University of Groningen & NL & $\mathrm{DE}$ & 17 \\
\hline 11 & Yale University & US & $\mathrm{EN}$ & 18 & 61 & University of Bristol & UK & EN & 20 \\
\hline 12 & University of California, Los Angeles & US & $\mathrm{EN}$ & 19 & 62 & University of Oslo & NO & WR & 19 \\
\hline 13 & University of Washington & US & EN & 19 & 63 & Uppsala University & SE & SV & 15 \\
\hline 14 & Cornell University & US & EN & 19 & 64 & University of California, Irvine & US & EN & 20 \\
\hline 15 & University of California, San Diego & US & $\mathrm{EN}$ & 20 & 65 & Aarhus University & DK & DA & 20 \\
\hline 16 & University College London & UK & EN & 19 & 66 & McMaster University & $\mathrm{CA}$ & $\mathrm{EN}$ & 19 \\
\hline 17 & University of Pennsylvania & US & $\mathrm{EN}$ & 18 & 67 & McGill University & $\mathrm{CA}$ & $\mathrm{EN}$ & 19 \\
\hline 18 & Johns Hopkins University & US & $\mathrm{EN}$ & 19 & 68 & University of Pittsburgh & US & $\mathrm{EN}$ & 18 \\
\hline 19 & ETH Zurich & $\mathrm{CH}$ & $\mathrm{DE}$ & 19 & 69 & École Normale Supérieure & FR & FR & 18 \\
\hline 22 & Northwestern University & US & EN & 19 & 72 & Peking University & $\mathrm{CN}$ & $\mathrm{ZH}$ & 19 \\
\hline 23 & University of Toronto & $\mathrm{CA}$ & EN & 19 & 73 & Erasmus University Rotterdam & NL & NL & 20 \\
\hline 24 & University of Michigan & US & $\mathrm{EN}$ & 19 & 74 & Rice University & US & EN & 20 \\
\hline 25 & University of Tokyo & JP & JA & 19 & 75 & Stockholm University & $\mathrm{SE}$ & SV & 19 \\
\hline 26 & Duke University & US & $\mathrm{EN}$ & 19 & 76 & École Polytechnique Fédérale de Lausanne & $\mathrm{CH}$ & $\mathrm{ZH}$ & 20 \\
\hline 27 & Imperial College London & UK & $\mathrm{EN}$ & 20 & 77 & Purdue University & US & $\mathrm{EN}$ & 19 \\
\hline 28 & University of Wisconsin-Madison & US & EN & 19 & 78 & Monash University & $\mathrm{AU}$ & EN & 20 \\
\hline 29 & New York University & US & $\mathrm{EN}$ & 19 & 79 & Rutgers University & US & $\mathrm{EN}$ & 18 \\
\hline 30 & University of Copenhagen & DK & DA & 15 & 80 & Boston University & US & $\mathrm{EN}$ & 19 \\
\hline 31 & University of British Columbia & $\mathrm{CA}$ & $\mathrm{EN}$ & 20 & 81 & Carnegie Mellon University & US & EN & 19 \\
\hline 32 & University of Edinburgh & UK & EN & 16 & 82 & Ohio State University & US & EN & 19 \\
\hline 33 & University of North Carolina at Chapel Hill & US & $\mathrm{EN}$ & 18 & 83 & University of Sydney & $\mathrm{AU}$ & EN & 19 \\
\hline 34 & University of Minnesota & US & EN & 19 & 84 & Nagoya University & JP & JA & 19 \\
\hline 35 & Kyoto University & JP & JA & 19 & 85 & Georgia Institute of Technology & US & EN & 19 \\
\hline 36 & Rockefeller University & US & $\mathrm{EN}$ & 20 & 86 & Pennsylvania State University & US & EN & 19 \\
\hline 37 & University of Illinois at Urbana-Champaign & US & $\mathrm{EN}$ & 19 & 87 & University of California, Davis & US & EN & 20 \\
\hline 38 & University of Manchester & UK & EN & 14 & 88 & Leiden University & NL & NL & 16 \\
\hline 39 & University of Melbourne & $\mathrm{AU}$ & $\mathrm{EN}$ & 19 & 89 & University of Florida & US & $\mathrm{EN}$ & 19 \\
\hline 40 & Pierre and Marie Curie University & FR & FR & 20 & 90 & KU Leuven & $\mathrm{BE}$ & FR & 15 \\
\hline 44 & Karolinska Institute & SE & SV & 19 & 94 & Technion - Israel Institute of Technology & IL & $\mathrm{HE}$ & 20 \\
\hline 45 & University of California, Santa Barbara & US & $\mathrm{EN}$ & 19 & 95 & University of Basel & $\mathrm{CH}$ & $\mathrm{ZH}$ & 15 \\
\hline 46 & King's College London & UK & $\mathrm{EN}$ & 16 & 96 & University of Göttingen & $\mathrm{DE}$ & $\mathrm{DE}$ & 18 \\
\hline 47 & Utrecht University & NL & NL & 17 & 97 & Australian National University & $\mathrm{AU}$ & EN & 20 \\
\hline 48 & The University of Texas Southwestern Medical Center at Dallas & US & $\mathrm{EN}$ & 20 & 98 & University of California, Santa Cruz & US & EN & 20 \\
\hline 49 & Tsinghua University & $\mathrm{CN}$ & $\mathrm{ZH}$ & 20 & 99 & Cardiff University & UK & EN & 19 \\
\hline 50 & Technical University of Munich & $\mathrm{DE}$ & $\mathrm{DE}$ & 19 & 100 & University of Arizona & US & EN & 19 \\
\hline
\end{tabular}



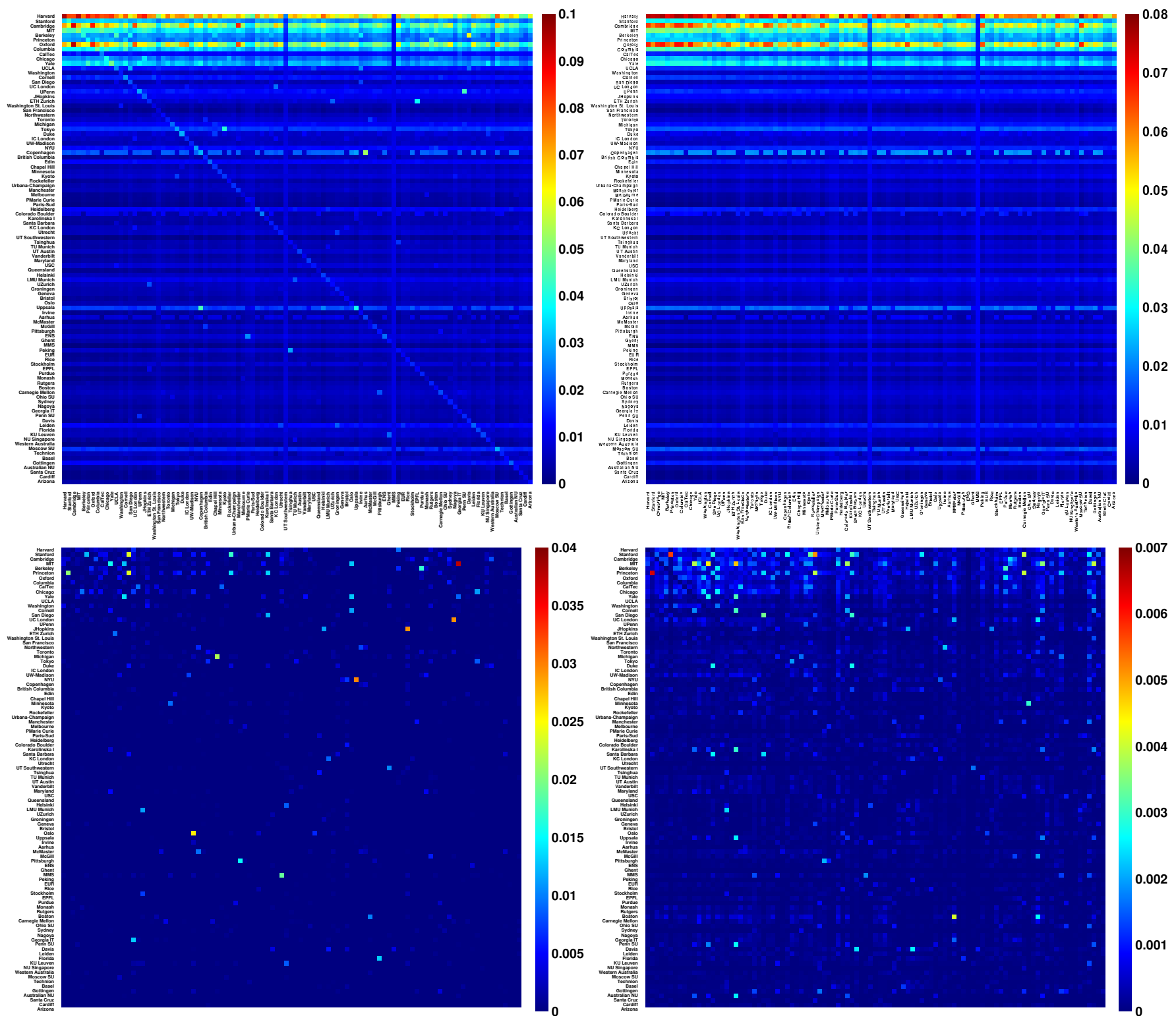

IPI

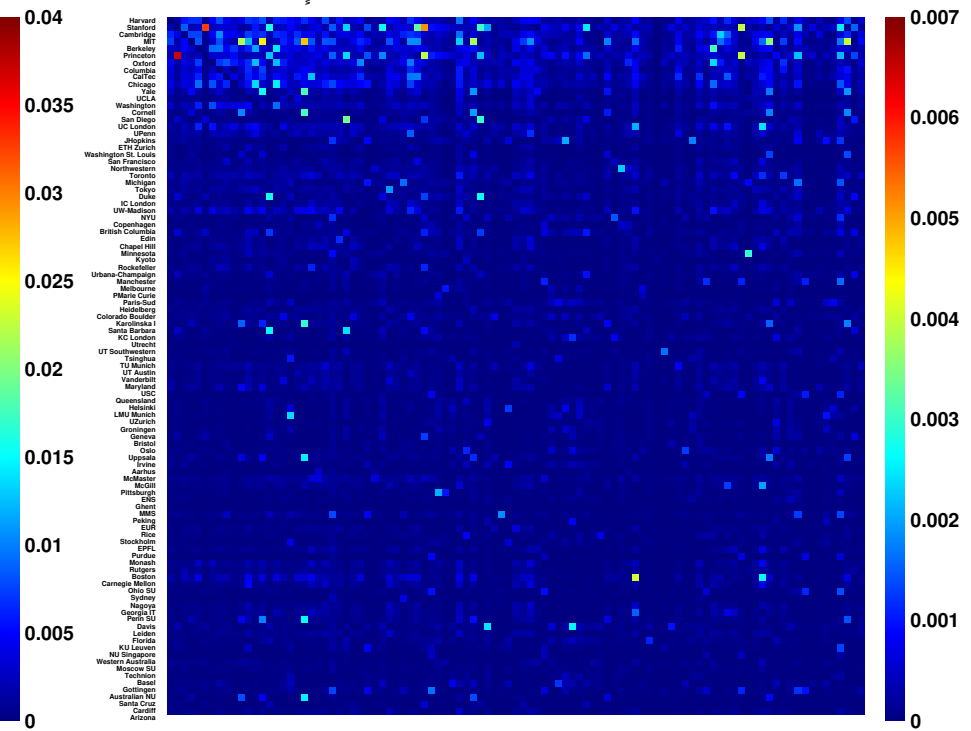

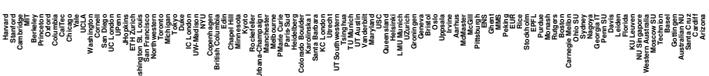

Fig. SI9. Reduced Google matrix $G_{\mathrm{R}}$ for top100 universities in ARWU (Tab. SI1) averaged over 24 Wikipedia editions. The full reduced Google matrix $G_{\mathrm{R}}$ is presented in top left panel, $G_{\mathrm{pr}}$ in top right panel, $G_{\mathrm{rr}}$ in bottom left panel, and $G_{\mathrm{qrnn}}$ in bottom right panel. The matrices weights are $W_{\mathrm{R}}=1, W_{\mathrm{pr}}=0.956, W_{\mathrm{rr}}=0.018$, and $W_{\mathrm{qr}}=0.026$. 
Table SI3. List of countries with corresponding country codes (CC) and language codes (LC). Only countries appearing in the top 100 universities of the 24 considered 2017 Wikipedia editions using PageRank, CheiRank, and 2DRank algorithms are listed here. LC is determined by the most spoken language in the given country. Country codes (CC) follow ISO 3166-1 alpha-2 standard [32]. Language codes are based on language edition codes of Wikipedia; WR represents all languages other than the considered 24 languages.

\begin{tabular}{|c|c|c|c|c|c|c|c|c|}
\hline $\mathrm{CC}$ & Country & LC & $\mathrm{CC}$ & Country & $\mathrm{LC}$ & $\mathrm{CC}$ & Country & $\mathrm{LC}$ \\
\hline $\mathrm{AE}$ & United Arab Emirates & $\mathrm{AR}$ & GE & Georgia & WR & $\mathrm{NP}$ & Nepal & WR \\
\hline $\mathrm{AF}$ & Afghanistan & FA & GL & Greenland & DA & NZ & New Zealand & EN \\
\hline $\mathrm{AL}$ & Albania & WR & GR & Greece & EL & $\mathrm{PE}$ & Peru & $\mathrm{ES}$ \\
\hline $\mathrm{AM}$ & Armenia & WR & GT & Guatemala & $\mathrm{ES}$ & PG & Papua New Guinea & EN \\
\hline $\mathrm{AO}$ & Angola & PT & HK & Hong Kong & $\mathrm{ZH}$ & $\mathrm{PH}$ & Philippines & EN \\
\hline $\mathrm{AR}$ & Argentina & ES & $\mathrm{HN}$ & Honduras & ES & PK & Pakistan & HI \\
\hline AT & Austria & $\mathrm{DE}$ & HR & Croatia & WR & PL & Poland & PL \\
\hline $\mathrm{AU}$ & Australia & EN & $\mathrm{HU}$ & Hungary & $\mathrm{HU}$ & PR & Puerto Rico & ES \\
\hline $\mathrm{AZ}$ & Azerbaijan & TR & ID & Indonesia & WR & PS & State of Palestine & $\mathrm{AR}$ \\
\hline BA & Bosnia and Herzegovina & WR & IE & Ireland & EN & $\mathrm{PT}$ & Portugal & $\mathrm{PT}$ \\
\hline $\mathrm{BD}$ & Bangladesh & WR & IL & Israel & $\mathrm{HE}$ & QA & Qatar & $\mathrm{AR}$ \\
\hline $\mathrm{BE}$ & Belgium & NL & IN & India & HI & $\mathrm{RO}$ & Romania & WR \\
\hline BG & Bulgaria & WR & IQ & Iraq & AR & $\mathrm{RS}$ & Serbia & WR \\
\hline BI & Burundi & WR & IR & Iran & FA & RU & Russia & $\mathrm{RU}$ \\
\hline BJ & Benin & FR & IS & Iceland & WR & SA & Saudi Arabia & $\mathrm{AR}$ \\
\hline $\mathrm{BN}$ & Brunei & MS & IT & Italy & IT & $\mathrm{SD}$ & Sudan & $\mathrm{AR}$ \\
\hline $\mathrm{BR}$ & Brazil & PT & JM & Jamaica & EN & $\mathrm{SE}$ & Sweden & SV \\
\hline BY & Belarus & RU & $\mathrm{JO}$ & Jordan & $\mathrm{AR}$ & SG & Singapore & $\mathrm{ZH}$ \\
\hline CA & Canada & EN & JP & Japan & JA & SK & Slovakia & WR \\
\hline $\mathrm{CD}$ & Dem. Rep. of Congo & FR & $\mathrm{KE}$ & Kenya & EN & SN & Senegal & FR \\
\hline $\mathrm{CH}$ & Switzerland & DE & KG & Kyrgyzstan & WR & SR & Suriname & NL \\
\hline CI & Ivory Coast & FR & $\mathrm{KH}$ & Cambodia & WR & SV & El Salvador & $\mathrm{ES}$ \\
\hline CL & Chile & ES & $\mathrm{KP}$ & North Korea & $\mathrm{KO}$ & SY & Syria & $\mathrm{AR}$ \\
\hline $\mathrm{CN}$ & China & $\mathrm{ZH}$ & $\mathrm{KR}$ & South Korea & $\mathrm{KO}$ & $\mathrm{TH}$ & Thailand & $\mathrm{TH}$ \\
\hline $\mathrm{CO}$ & Colombia & ES & KW & Kuwait & $\mathrm{AR}$ & $\mathrm{TL}$ & Timor-Leste & $\mathrm{PT}$ \\
\hline $\mathrm{CR}$ & Costa Rica & $\mathrm{ES}$ & $\mathrm{KZ}$ & Kazakhstan & WR & $\mathrm{TN}$ & Tunisia & $\mathrm{AR}$ \\
\hline $\mathrm{CU}$ & Cuba & $\mathrm{ES}$ & LA & Laos & WR & TR & Turkey & TR \\
\hline $\mathrm{CV}$ & Cape Verde & $\mathrm{PT}$ & LB & Lebanon & $\mathrm{AR}$ & TW & Taiwan & $\mathrm{ZH}$ \\
\hline CY & Cyprus & EL & LT & Lithuania & WR & UA & Ukraine & WR \\
\hline $\mathrm{CZ}$ & Czech Republic & WR & LV & Latvia & WR & UG & Uganda & EN \\
\hline $\mathrm{DE}$ & Germany & $\mathrm{DE}$ & LY & Libya & $\mathrm{AR}$ & UK & United Kingdom & EN \\
\hline DK & Denmark & DA & MA & Morocco & $\mathrm{AR}$ & US & United States & $\mathrm{EN}$ \\
\hline $\mathrm{DO}$ & Dominican Republic & ES & MK & Macedonia & WR & UY & Uruguay & $\mathrm{ES}$ \\
\hline DZ & Algeria & $\mathrm{AR}$ & MM & Myanmar & WR & UZ & Uzbekistan & WR \\
\hline $\mathrm{EC}$ & Ecuador & ES & MT & Malta & EN & VA & Holy See & IT \\
\hline $\mathrm{EE}$ & Estonia & WR & MX & Mexico & $\mathrm{ES}$ & $\mathrm{VE}$ & Venezuela & ES \\
\hline EG & Egypt & $\mathrm{AR}$ & MY & Malaysia & MS & $\mathrm{VN}$ & Vietnam & VI \\
\hline $\mathrm{ES}$ & Spain & ES & $\mathrm{NE}$ & Niger & FR & YE & Yemen & $\mathrm{AR}$ \\
\hline FI & Finland & WR & NG & Nigeria & EN & $\mathrm{ZA}$ & South Africa & WR \\
\hline $\mathrm{FO}$ & Faroe Islands & DA & NL & Netherlands & NL & ZW & Zimbabwe & EN \\
\hline FR & France & FR & $\mathrm{NO}$ & Norway & WR & & & \\
\hline
\end{tabular}


Table SI4. List of 240 countries and territories ranked from 2017 Wikipedia English edition using PageRank algorithm. Country codes (CC) follow ISO 3166-1 alpha-2 standard [32].

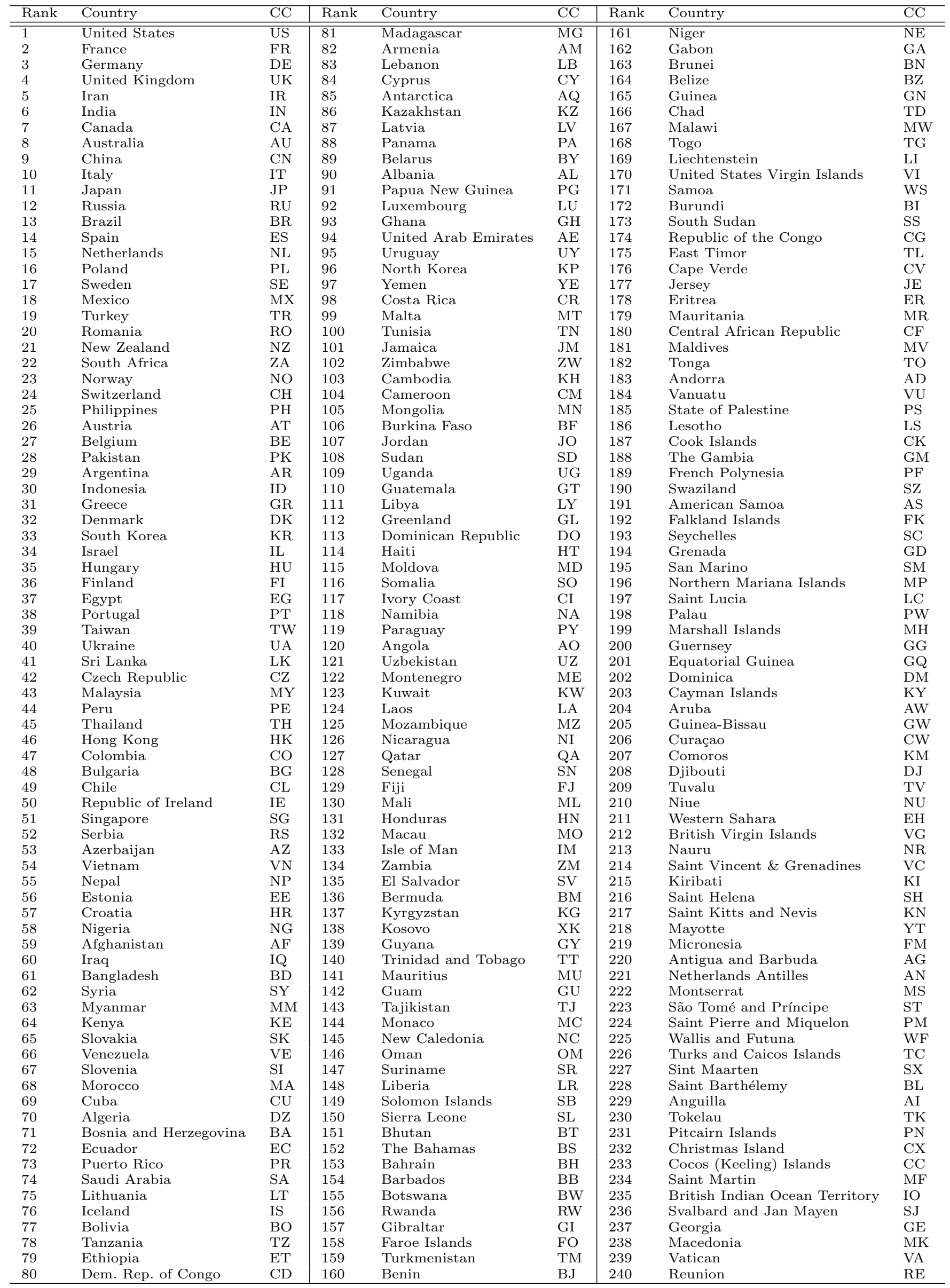


Table SI5. Universities from top100 2017 ARWU ordered according to PageRank algorithm applied to the reduced Google matrix averaged over 24 Wikipedia editions.

\begin{tabular}{|c|c|c|c|c|c|}
\hline Rank & PageRank & University & Rank & PageRank & University \\
\hline 1 & 0.0745472 & Harvard University & 51 & 0.00583189 & University of Washington \\
\hline 2 & 0.0621785 & University of Oxford & 52 & 0.00560402 & Northwestern University \\
\hline 3 & 0.0609665 & University of Cambridge & 53 & 0.00556462 & University of Groningen \\
\hline 4 & 0.0397826 & $\mathrm{MIT}^{a}$ & 54 & 0.00545821 & University of Wisconsin-Madison \\
\hline 5 & 0.0355868 & Columbia University & 55 & 0.00545373 & $\mathrm{U}$ of I, Illinois $^{f}$ \\
\hline 6 & 0.0333098 & Yale University & 56 & 0.00541549 & Boston University \\
\hline 7 & 0.0306724 & Stanford University & 57 & 0.00539776 & University of Oslo \\
\hline 8 & 0.0282621 & $\mathrm{UC}$ Berkeley ${ }^{b}$ & 58 & 0.00530861 & University of Geneva \\
\hline 9 & 0.0266544 & Princeton University & 59 & 0.00516361 & Ohio State University \\
\hline 10 & 0.0250087 & University of Chicago & 60 & 0.00502027 & Tsinghua University \\
\hline 11 & 0.0195607 & University of Copenhagen & 61 & 0.00491138 & Ghent University \\
\hline 12 & 0.0189782 & Uppsala University & 62 & 0.0049034 & University of Arizona \\
\hline 13 & 0.0176773 & University of Tokyo & 63 & 0.00473034 & University of California, San Diego \\
\hline 14 & 0.0165834 & Moscow State University & 64 & 0.00468699 & Purdue University \\
\hline 15 & 0.0148824 & Cornell University & 65 & 0.00465378 & University of Basel \\
\hline 16 & 0.0145334 & University of Pennsylvania & 66 & 0.0046221 & UNC Chapel Hill $^{g}$ \\
\hline 17 & 0.0141382 & $\mathrm{UCLA}^{c}$ & 67 & 0.00455207 & Technical University of Munich \\
\hline 18 & 0.0131496 & New York University & 68 & 0.00444405 & University of Sydney \\
\hline 19 & 0.0131307 & California Institute of Technology & 69 & 0.00437431 & University of Maryland, College Park \\
\hline 20 & 0.0125402 & University Göttingen & 70 & 0.00430464 & Rutgers University \\
\hline 21 & 0.0124228 & Leiden University & 71 & 0.00404074 & Karolinska Institute \\
\hline 22 & 0.0124135 & Heidelberg University & 72 & 0.0039799 & University of Pittsburgh \\
\hline 23 & 0.012155 & University of Edinburgh & 73 & 0.00396299 & Georgia Institute of Technology \\
\hline 24 & 0.0115044 & University of Michigan & 74 & 0.00385092 & Penn State University \\
\hline 25 & 0.0107339 & Johns Hopkins University & 75 & 0.00369518 & Washington University in St. Louis \\
\hline 26 & 0.0105176 & University of Munich $^{d}$ & 76 & 0.00364923 & University of British Columbia \\
\hline 27 & 0.00917197 & University College London & 77 & 0.00360777 & Australian National University \\
\hline 28 & 0.00872849 & Duke University & 78 & 0.00355039 & University of California, Santa Barbara \\
\hline 29 & 0.00819352 & University of Southern California & 79 & 0.00352429 & University of Bristol \\
\hline 30 & 0.00814739 & ETH Zurich & 80 & 0.00337135 & National University of Singapore \\
\hline 31 & 0.00802171 & Kyoto University & 81 & 0.00326108 & Rockefeller University \\
\hline 32 & 0.00797102 & Aarhus University & 82 & 0.00320465 & Rice University \\
\hline 33 & 0.00795232 & University of Colorado Boulder & 83 & 0.00311399 & University of Melbourne \\
\hline 34 & 0.00783698 & École Normale Supérieure & 84 & 0.00307731 & Vanderbilt University \\
\hline 35 & 0.00766772 & Peking University & 85 & 0.00290042 & Erasmus University Rotterdam \\
\hline 36 & 0.0076523 & Stockholm University & 86 & 0.00284488 & University of California, Davis \\
\hline 37 & 0.00744185 & University Toronto & 87 & 0.00279911 & $\mathrm{EPFL}^{h}$ \\
\hline 38 & 0.0070079 & University of Texas at Austin & 88 & 0.00273436 & Pierre and Marie Curie University \\
\hline 39 & 0.00685167 & Imperial College London & 89 & 0.00272264 & Nagoya University \\
\hline 40 & 0.00681529 & Utrecht University & 90 & 0.00265046 & University of California, Irvine \\
\hline 41 & 0.00658785 & University of Minnesota & 91 & 0.00263142 & University of Queensland \\
\hline 42 & 0.00654586 & Carnegie Mellon University & 92 & 0.00210331 & University of California, San Francisco \\
\hline 43 & 0.00653689 & University of Helsinki & 93 & 0.0021006 & Monash University \\
\hline 44 & 0.00651107 & KU Leuven & 94 & 0.00198778 & University of California, Santa Cruz \\
\hline 45 & 0.0064442 & King's College London & 95 & 0.00189623 & Cardiff University \\
\hline 46 & 0.00639753 & University of Zurich & 96 & 0.00186566 & McMaster University \\
\hline 47 & 0.00623053 & McGill University & 97 & 0.00186277 & University of Paris-Sud \\
\hline 48 & 0.00621359 & University of Manchester & 98 & 0.00157434 & The University of Western Australia \\
\hline 49 & 0.00602113 & University of Florida & 99 & 0.0011675 & UT Southwestern ${ }^{i}$ \\
\hline 50 & 0.00600767 & Technion $^{e}$ & 100 & 0.00102057 & Mayo Medical School \\
\hline
\end{tabular}

${ }^{a}$ Massachusetts Institute of Technology, ${ }^{b}$ University of California, Berkeley, ${ }^{c}$ University of California, Los Angeles, ${ }^{d}$ Ludwig Maximilian University of Munich, ${ }^{e}$ Technion - Israel Institute of Technology, ${ }^{f}$ University of Illinois Urbana-Champaign, ${ }^{g}$ University of North Carolina at Chapel Hill, ${ }^{h}$ École Polytechnique Fédérale de Lausanne, ${ }^{i}$ The University of Texas Southwestern Medical Center at Dallas 


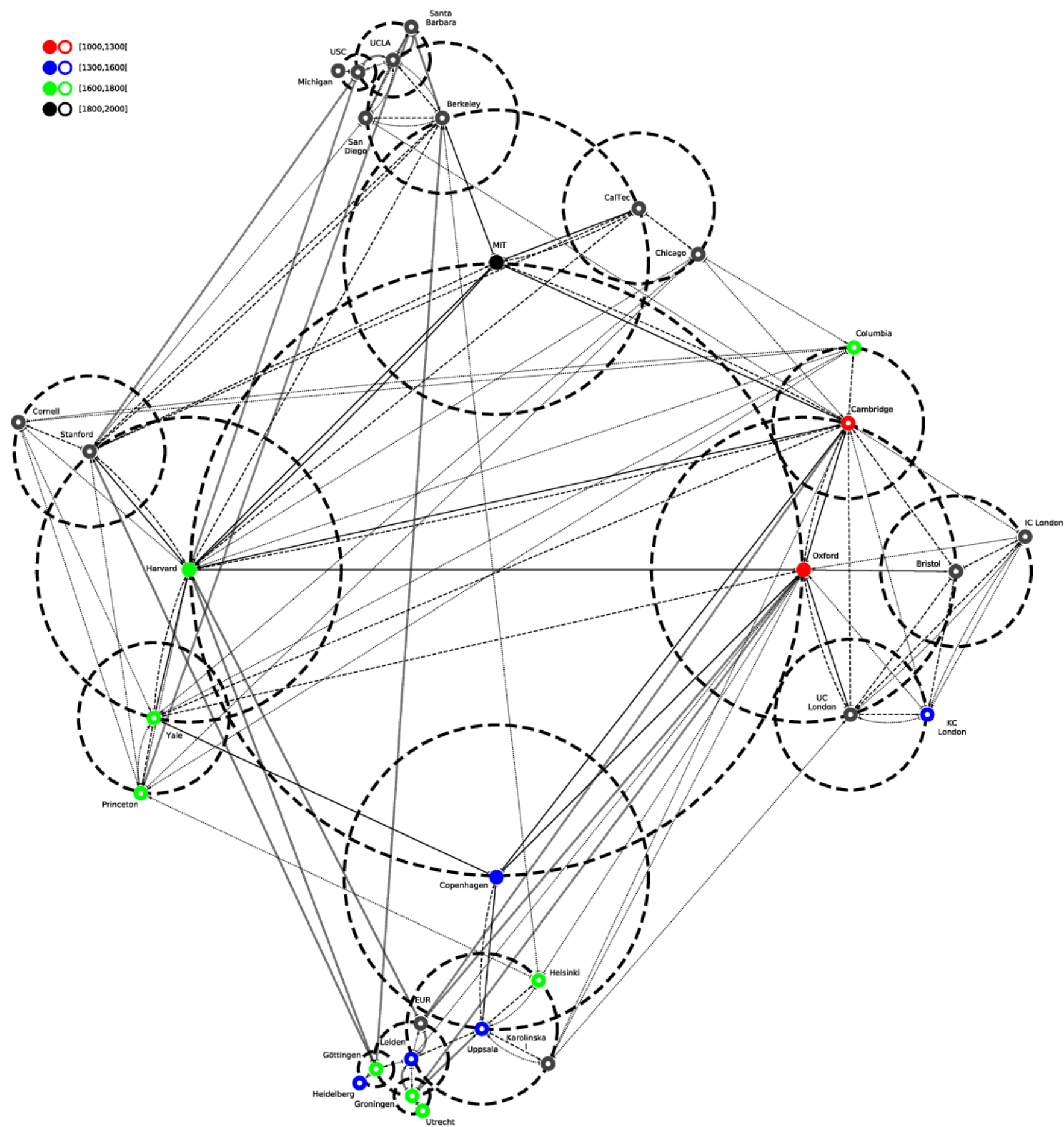

Fig. SI10. Reduced network constructed for universities listed in Tab. SI5 computed from $G_{\mathrm{rr}}+G_{\mathrm{qrnd}}$ averaged over 24 Wikipedia editions. Color filled nodes are time period leaders. We obtain 4 friendship levels with corresponding links: black solid lines (level 1), dashed lines (level 2), doted lines (level 3) and " $"$ symbol lines (4+). 


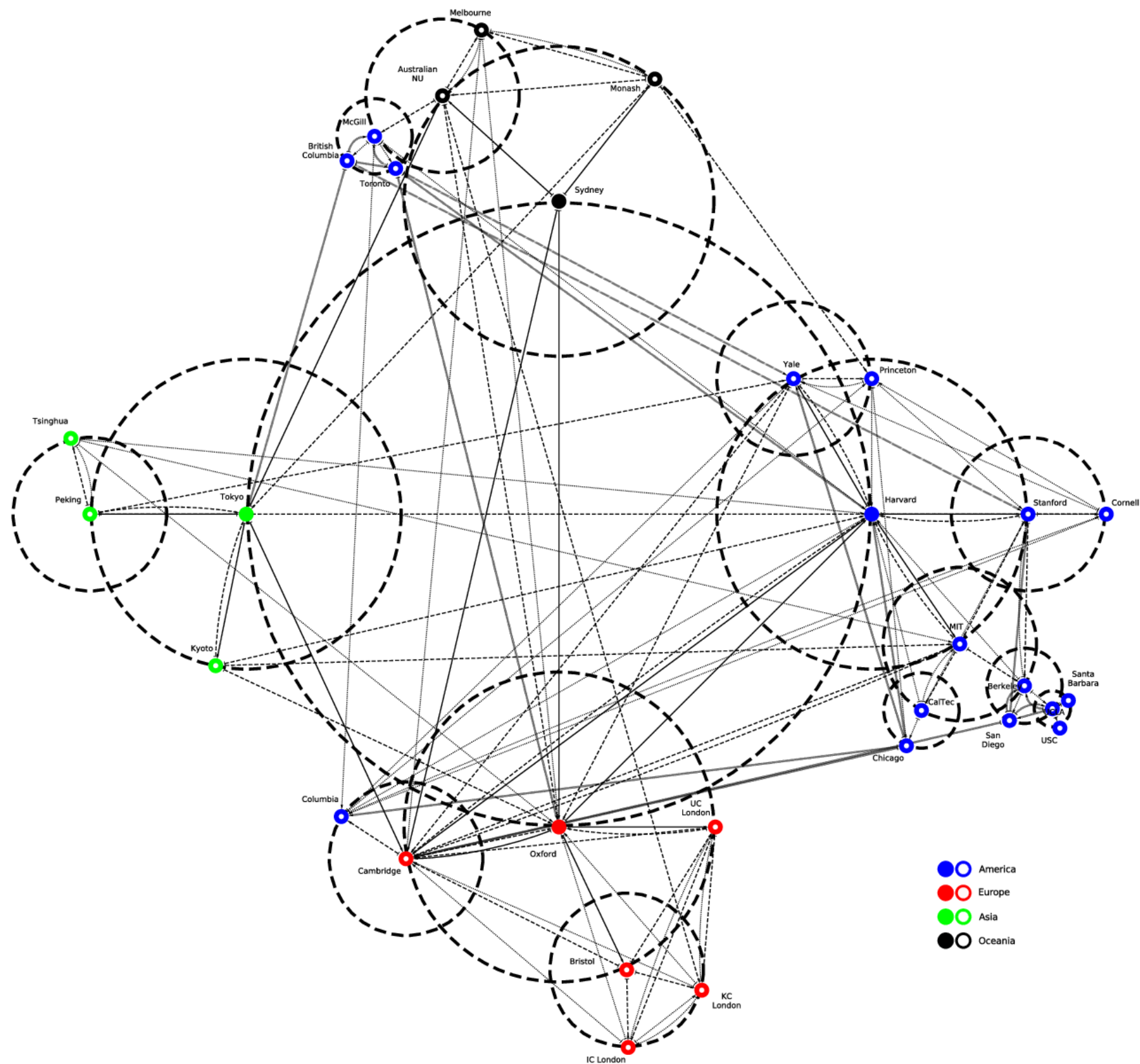

Fig. SI11. Reduced network constructed for universities listed in Tab. SI5 computed from $G_{\text {rr }}+G_{\text {qrnd }}$ averaged over 24 Wikipedia editions. Color filled nodes are continent leaders. We obtain 4 friendship levels with corresponding links: black solid lines (level 1), dashed lines (level 2), doted lines (level 3) and "\" symbol lines (4+). 